\title{
VOLUME OF LINE BUNDLES VIA VALUATION VECTORS (DIFFERENT FROM OKOUNKOV BODIES)
}

\author{
O. BRAUNLING
}

\begin{abstract}
Up to a factor $1 / n$ !, the volume of a big line bundle agrees with the Euclidean volume of its Okounkov body. The latter is the convex hull of top rank valuation vectors of sections, all with respect to a single flag. In this text we give a different volume formula, valid in the ample cone, also based on top rank valuation vectors, but mixing data along several different flags.
\end{abstract}

A recent paper of Küronya and Lozovanu uses an interesting viewpoint to study NewtonOkounkov bodies $\Delta_{\underline{v}}(D)$ of surfaces: The valuation vectors whose convex hull forms $\Delta_{\underline{v}}(D)$ can be interpreted as local intersection numbers, KL18, Remark 1.8]. This idea is the starting point for this text. A priori unrelated to this, work of Parshin expresses global intersection numbers in terms of rank $n$ valuation vectors Par83. In this paper we combine both ideas. We get a new formula for the volume of $D$, using valuation vectors belonging to a number of different valuations/flags. As such, it is of a quite different nature than the construction of Okounkov bodies, which uses only a single valuation. Pursuing this idea, we are led to a formula whose summands all have the shape " $\frac{1}{n !} \operatorname{det}(. .$.$) ", which has a very tempting$ interpretation as the volume of an $n$-simplex. Although it remains an unresolved mystery, this suggests that our formula might also have an interpretation in terms of convex polytopes - albeit necessarily of a different nature than Okounkov bodies. by

Let us explain this a little slower and more carefully: If $\underline{x}^{1}, \ldots, \underline{x}^{n} \in \mathbb{R}^{n}$ are vectors, then

$$
\text { simplex }\left\langle\underline{x}^{1}, \underline{x}^{2}, \ldots, \underline{x}^{n}\right\rangle
$$

we refer to the oriented simplex formed as the convex hull of $\left\{0, \underline{x}^{1}, \underline{x}^{2}, \ldots, \underline{x}^{n}\right\}$. We also remember the orientation, i.e. whether $\underline{x}^{1}, \underline{x}^{2}, \ldots, \underline{x}^{n}$ define the standard orientation or not. It has a (signed) volume,

$$
\text { Vol simplex }\left\langle\underline{x}^{1}, \underline{x}^{2}, \ldots, \underline{x}^{n}\right\rangle=\frac{1}{n !} \operatorname{det}\left[\underline{x}^{i}\right]_{i=1, \ldots, n} .
$$

Any divisor $D$ on an irreducible $n$-dimensional smooth projective variety $X / k$ has an invariant called its 'volume'. This invariant is usually defined in terms of the growth of global sections under taking powers of the attached line bundle. Over $\mathbb{C}$, and if $D$ happens to be very ample, one can alternatively pull back the Fubini-Study metric of $\mathbb{P}_{\mathbb{C}}^{N}$ along the associated projective embedding

$$
X \hookrightarrow \mathbb{P}_{\mathbb{C}}^{N}
$$

and then the Riemannian metric volume of $X$ can also be taken as the definition, at least after rescaling it by the factor $\frac{1}{n !}$. However, the concept of 'volume' can also be understood

The author was partially supported by DFG GK1821 "Cohomological Methods in Geometry". 
as the real volume of a convex body in Euclidean space. To do this, pick a top rank valuation $\underline{v}: k(X)^{\times} \rightarrow \mathbb{Z}_{\text {lex }}^{n}$ on the function field of $X$, and define

$$
\Delta_{\underline{v}}(D):=\text { closed convex hull }\left\{\frac{1}{m} \underline{v}(s)\right\}_{s, m} \quad \text { inside } \mathbb{R}^{n},
$$

where $(s, m)$ runs through all pairs $m \geq 1$ and $s \in H^{0}\left(X, \mathcal{O}_{X}(m D)\right) \backslash\{0\}$. This is the socalled Newton-Okounkov body of $D$ for the valuation $\underline{v}$. Changing the valuation gives different convex bodies $\Delta_{(-)}(D)$, but they always have the same volume. This volume is again $\frac{1}{n !}$-th of the birational 'volume'. This characterization of volume is due to Lazarsfeld and Mustaţă LM09.

Let us focus on a rather special situation: Assume that $D$ is ample and that the so-called graded semigroup $\Gamma_{v}(D)$ is finitely generated (we define this carefully later). Then we can phrase the above fact in a stronger form: For $m \gg 1$ big enough to make $m D$ very ample, we get a local trivialization $\left(U_{\alpha}, h_{\alpha}\right)_{\alpha \in I}$ of $m D$ as a Cartier divisor on a finite open cover $\left(U_{\alpha}\right)_{\alpha \in I}$; each $h_{\alpha}$ comes from a global section of $\mathcal{O}_{X}(m D)$, and moreover

$$
\Delta_{\underline{v}}(D)=\text { convex hull }\left\{\frac{1}{m} \underline{v}\left(h_{\alpha}\right)\right\}_{\alpha \in I} .
$$

In particular, in this case $\Delta_{\underline{v}}(D)$ is a convex polytope. By a 'local trivialization as a Cartier divisor' we mean an explicit Čech 0-cocycle $\check{H}^{0}\left(\left\{U_{\alpha}\right\}, \mathcal{K}_{X}^{\times} / \mathcal{O}_{X}^{\times}\right)$. Note that we take the convex hull running over local equations $h_{\alpha}$, but all with respect to the same valuation. Our main result gives a different formula for the volume of $D$. It

(A) also uses only the global sections $h_{\alpha}$ as above, but

(B) with respect to a family of different top rank valuations $\underline{w}$, depending on the global geometry of the variety.

Main Theorem. Let $X / k$ be an irreducible smooth projective variety of dimension $n$.

(1) Let $\underline{v}: k(X)^{\times} \rightarrow \mathbb{Z}_{\text {lex }}^{n}$ be a top rank valuation.

(2) Let $D$ be an ample divisor.

(3) Suppose the graded semigroup $\Gamma_{\underline{v}}(D)$ is finitely generated.

(4) Choose $m \geq 1$ such that $m D$ is very ample.

Then there is a local trivialization of $m D$ as a Cartier divisor, $\left(U_{\alpha}, h_{\alpha}\right)_{\alpha \in I}$ such that the $h_{\alpha}$ are restrictions of global sections to the opens $U_{\alpha}$. Moreover,

$$
\begin{aligned}
\operatorname{Vol}\left(\underset{\alpha \in I}{\operatorname{convex}} \operatorname{hull}\left(\frac{1}{m} \underline{v}\left(h_{\alpha}\right)\right)\right) & =\sum_{\underline{w}} \sum_{c=0}^{n}(-1)^{c}[k(\underline{w}): k] \\
& \text { Vol simplex }\left\langle\underline{w}\left(h_{\alpha_{0}(\underline{w})}\right), \ldots \underline{w} \widehat{\left(h_{\alpha_{c}(\underline{w})}\right)} \ldots, \underline{w}\left(h_{\alpha_{n}(\underline{w})}\right)\right\rangle .
\end{aligned}
$$

Here $\underline{w}$ runs over a finite set of top rank valuations $\underline{w}: k(X)^{\times} \rightarrow \mathbb{Z}_{\text {lex }}^{n}$ and there are welldefined values $\alpha_{i}(\underline{w}) \in I$ attached to each. We write $k(\underline{w})$ to denote the residue field of $\underline{w}$. Both sides of the equation agree with the volume of the Newton-Okounkov body.

This is a shortened formulation of our Theorem 3.17. The full version of the theorem explains where the valuations $\underline{w}$ and the values $\alpha_{i}(\underline{w})$ come from and how to determine them. It turns out that they will be canonically determined once any trivialization with such properties is chosen. However, the full story is somewhat involved (it involves the set $\mathcal{G}$ of Definition 3.11 if you wish to jump ahead).

Note that we have the global sections $h_{(-)}$on both sides of the equation, but only the chosen fixed valuation $\underline{v}$ on the left, while several different valuations appear on the right. It 
is too complicated to describe here how the $\underline{w}$ arise, but their choice is independent of the choice of $\underline{v}$. In particular, in a general situation, $\underline{v}$ will not appear among the $\underline{w}$ on the right side.

The statement of the main theorem is quite convoluted, so let us stress the main point informally:

Slogan. If $D$ is very ample, there is a formula

Volume of Okounkov body $=\mathbb{Z}$-linear comb. of volumes of $n$-simplices

$$
\begin{aligned}
\text { convex hull of } \underline{v}\left(h_{(-)}\right) \quad \text { simplices with vertices at } \underline{w}\left(h_{(-)}\right) \\
\text {for a finite number of valuations } \underline{w},
\end{aligned}
$$

where the $h_{(-)}$are global sections which restrict on opens of a finite open cover to local trivializations of $D$ as a Cartier divisor.

We will explicitly evaluate both sides of our formula in a family of examples on Hirzebruch surfaces. Just to get into the "look \& feel" how our formula may look in a concrete example case:

$$
\begin{aligned}
& \operatorname{Vol}\left(\text { convex hull }\left(\underline{v}\left(h_{0}\right) ; \underline{v}\left(h_{2}\right) ; \underline{v}\left(h_{4}\right) ; 0\right)\right)= \\
& \quad \frac{1}{2} \operatorname{det}\left(\begin{array}{ll}
\underline{w}_{1}\left(h_{4}\right) & \underline{w}_{1}\left(h_{2}\right) \\
\underline{w}_{2}\left(h_{4}\right) & \underline{w}_{2}\left(h_{2}\right)
\end{array}\right)-\frac{1}{2} \operatorname{det}\left(\begin{array}{ll}
\underline{w}_{1}\left(h_{0}\right) & \underline{w}_{1}\left(h_{2}\right) \\
\underline{w}_{2}\left(h_{0}\right) & \underline{w}_{2}\left(h_{2}\right)
\end{array}\right) \\
& \quad+\frac{1}{2} \operatorname{det}\left(\begin{array}{ll}
\underline{w}_{1}\left(h_{0}\right) & \underline{w}_{1}\left(h_{4}\right) \\
\underline{w}_{2}\left(h_{0}\right) & \underline{w}_{2}\left(h_{4}\right)
\end{array}\right)-\frac{1}{2} \operatorname{det}\left(\begin{array}{ll}
\underline{w}_{1}^{\prime}\left(h_{0}\right) & \underline{w}_{1}^{\prime}\left(h_{4}\right) \\
\underline{w}_{2}^{\prime}\left(h_{0}\right) & \underline{w}_{2}^{\prime}\left(h_{4}\right)
\end{array}\right) .
\end{aligned}
$$

We refer to $\$ 4$ for notation and details. Three top rank valuations $\underline{v}, \underline{w}$ and $\underline{w}^{\prime}$ appear in this formula.

We have chosen a particularly provocative formulation in Equation 0.3 . In principle we are only comparing volumes, so we could also have spelled out the volume of the simplices on the right-hand side. However, since the vertices entering the convex geometry on the left and right side look so similar, I would dream that this equality might just be a "shadow" of a stronger identity. For example, writing

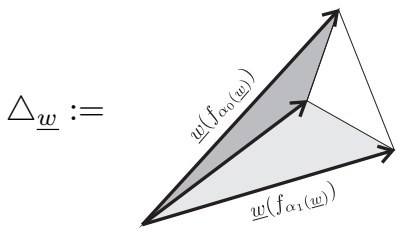

for the $n$-simplices on the right-hand side of the formula, one could imagine that there might be a systematic way to assemble these simplices to a polytope in their own right. So far all my attempts to properly understand this have been futile, so this remains a wild dream.

The right hand side of the formula in the theorem is also valid without the assumption that $\Gamma_{\underline{v}}(D)$ is finitely generated. However, then the Newton-Okounkov body does not need to be a polytope and we lose the left side of the equation. We still get the correct volume though (this will be Theorem 3.15).

Our method is based on not very well-known approaches to intersection theory, notably mixing ideas of Bloch [Blo74] and Parshin [Par83, [PF99]. These approaches have a special strength: they do not have any trouble if the set-theoretic dimension of the intersection is wrong, e.g. for negative self-intersections, and at the same time they remain local (unlike 
Riemann-Roch style ideas, like Snapper, Kleiman's via $\chi$ ) and algebraic, unlike for example singular cohomology. Concretely, we use the framework of Bloch, which uses Čech cohomology instead of the less well-known adèles of schemes in Parshin's approach. Nonetheless, the ideas how this relates to top rank valuations are imported from Parshin's method. We wrote this text in a self-contained way, so no previous familiarity with these methods is assumed.

In this text a 'variety' is a scheme which is finite type and separated over some field. Natural numbers begin with zero, $\mathbb{N}:=\mathbb{Z}_{\geq 0}$.

\section{VALUATION VECTORS AND OKOUNKOV BOdY}

Let $k$ be a field. Suppose $X / k$ is an $n$-dimensional irreducible smooth projective variety. Let $v$ be a top rank valuation on $X$ (i.e. of rank equal to the dimension $n$ ). It is necessarily discrete with (the lexicographically ordered) value group $\mathbb{Z}_{\text {lex }}^{n}$, see [ZS75, Ch. VI, p. 90, Corollary]; we write $\underline{v}: k(X)^{\times} \rightarrow \mathbb{Z}_{\text {lex }}^{n}$. We also call $\underline{v}(f)=\left(\underline{v}_{1}(f), \ldots, \underline{v}_{n}(f)\right) \in \mathbb{R}_{\text {lex }}^{n}$ a valuation vector and the $\underline{v}_{i}(f) \in \mathbb{Z}$ its components. We shall always tacitly assume that our valuations are trivial on the base field $k$. One way to produce such a valuation is to pick an admissible flag:

Definition 1.1. A flag in $X$ is the datum

$$
Y_{\bullet}: X=Y_{0} \supset Y_{1} \supset Y_{2} \supset \cdots \supset Y_{0},
$$

where $Y_{i}$ is an integral closed subscheme of codimension $i$. In particular, $Y_{0}$ is a single closed point. Following [LM09], we call the flag admissible if each $Y_{i}$ is regular at $Y_{0}$.

While LM09 focusses on admissible flags, the entire theory of Newton-Okounkov bodies can be formulated with respect to arbitrary top rank valuations on the rational function field of a variety. This is very nicely explained in $\mathrm{CFK}^{+} 17$.

The graded semigroup attached to a divisor $D$ is

$$
\Gamma_{\underline{v}}(D):=\left\{(\underline{v}(s), m) \mid s \in H^{0}\left(X, \mathcal{O}_{X}(m D)\right) \backslash\{0\} \text { for any } m \geq 0\right\} \subseteq \mathbb{Z}^{n} \times \mathbb{N} .
$$

Definition 1.2. The (Newton-) Okounkov body $\Delta_{v}(D) \subset \mathbb{R}^{n}$ is given by

$$
\Delta_{\underline{v}}(D):=\overline{\text { convex hull }\left(\Gamma_{\underline{v}}(D)\right)} \cap\left(\mathbb{R}^{n} \times\{1\}\right),
$$

i.e. we take the closed convex hull of all the points in $\Gamma_{\underline{v}}(D)$ inside $\mathbb{R}^{n} \times \mathbb{R}$, and then intersect with the coordinate hyperplane $m=1$. Alternatively,

$$
\Delta_{\underline{v}}(D):=\overline{\text { convex hull }\left\{\frac{1}{m} \underline{v}(s) \mid s \in H^{0}\left(X, \mathcal{O}_{X}(m D)\right) \backslash\{0\} \text { for } m \geq 1\right\}}
$$

inside $\mathbb{R}^{n}$.

History 1.3. The original definition is due to Okounkov Oko96, Oko03, but with a focus on a different kind of question. Both [LM09] as well as KK12 have brought the concept to the midst of birational geometry.

For $m=0$ observe that $\Gamma_{v}(D)$ contains the origin $(0, \ldots, 0)$, so as soon as it contains the point $(\underline{v}(s), m)$, the connecting segment to the origin crosses the $m=1$ hyperplane at $\left(\frac{1}{m} \underline{v}(s), 1\right)$. This essentially shows the equivalence of both definitions.

Example 1.4. If the graded semigroup $\Gamma_{\underline{v}}(D)$ is finitely generated, $\Delta_{\underline{v}}(D)$ is a rational polytope. Examples with $\Gamma_{\underline{v}}(D)$ finitely generated are rather rare, but see AKL14, Proposition 14] for a construction of examples.

We begin with an elementary observation. 
Proposition 1.5. Let $X / k$ be an irreducible smooth projective variety, $\underline{v}$ a top rank valuation and $D$ an ample divisor. Pick $m \geq 1$ such that $m D$ is very ample. Then there exists a local trivialization $\left(U_{\alpha}, h_{\alpha}\right)_{\alpha \in I}$ of $\mathcal{O}_{X}(m D)$ as a Cartier divisor with I a finite index set and

(1) each $h_{\alpha}$ on $U_{\alpha}$ is the restriction of a global section; for simplicity we write $h_{\alpha} \in$ $H^{0}\left(X, \mathcal{O}_{X}(m D)\right)$;

(2) $\left(h_{\alpha}\right)_{\alpha}$ with $h_{\alpha} \in H^{0}\left(U_{\alpha}, \mathcal{K}_{X}^{\times} / \mathcal{O}_{X}^{\times}\right)$is a Cartier divisor representative of $m D$;

(3) and moreover the trivialization polytope

$$
\underset{\alpha \in I}{\operatorname{convex}} \operatorname{hull}\left(\frac{1}{m} \underline{v}\left(h_{\alpha}\right)\right) \subseteq \Delta_{\underline{v}}(D),
$$

is contained inside the Newton-Okounkov body.

Proof. Direct. Once $m D$ is very ample, it is globally generated.

Example 1.6. In general the polytope of Equation 1.3 will be strictly smaller than $\Delta_{v}(D)$. Necessarily so, because the construction always yields a polytope, yet there are examples of Newton-Okounkov bodies which are non-polyhedral, LM09, §6.3]. Such examples can only exist in dimension $\geq 3$, and further such examples can be found in [KLM12, §3].

Example 1.7. Quite on the contrary, for an ample divisor on a toric variety and $\underline{v}$ coming from a $T$-invariant flag, we can achieve equality in Equation 1.3. Indeed, the Newton-Okounkov body always agrees with the divisor polytope, so it is necessarily polyhedral, as follows from [LM09, Proposition 6.1 , (i)]. Let $X / k$ be a smooth proper toric variety. Let $D$ be any $T$-invariant divisor, i.e.

$$
D=\sum_{\sigma} n_{\sigma} D_{\sigma}
$$

where $\sigma \in \Sigma(1)$ runs through the rays of the polyhedral fan $\Sigma \subset N_{\mathbb{R}}$ such that $X=X(\Sigma)$. Following the notation of Fulton $\left[\right.$ Ful93, let $M:=\operatorname{Hom}(N, \mathbb{Z})$. Then if $u_{\alpha}$ runs through the maximal cones of $\Sigma$, on each affine open $U_{\alpha}:=\operatorname{Spec} k\left[u_{\alpha}^{\vee} \cap M\right]$ the line bundle $\mathcal{O}(D)$ is trivialized $\left.\mathcal{O}(D)\right|_{U_{\alpha}}=\left.\frac{1}{h_{\alpha}} \mathcal{K}_{X}\right|_{U_{\alpha}}$ by the unique monomial $h_{\alpha} \in M$ determined by

$$
\left\langle h_{\alpha}, \sigma\right\rangle=-n_{\sigma},
$$

where $\sigma$ runs through all rays of the cone $u_{\alpha}$. As $X$ is smooth, and thus $\sigma$ a smooth cone, this amounts to a system of equations with a unique solution. We have

$$
\left.H^{0}\left(X, \mathcal{O}_{X}(D)\right)=k \text { - } \operatorname{span}\langle h \in M|\langle h, \sigma\rangle \geq-n_{\sigma} \text { for all } \sigma \in \Sigma(1)\right\rangle,
$$

the usual description of the sections via the divisor polytope $P_{D}$, [Ful93, p. 66]. As is discussed in the proof of [Ful93, p. 68, Proposition], $\mathcal{O}(D)$ is generated by global sections if and only if for each $\alpha,\langle h, \sigma\rangle \geq-n_{\sigma}$ holds for all rays $\sigma$ (also those not being faces of the cone $u_{\alpha}$ ). If $D$ is ample, this is satisfied, [Ful93, p. 70, Proposition]. This puts us in the situation described in Proposition 1.5. but with equality of polytopes in Equation 1.3 . We depict below on the left the polyhedral fan in $N_{\mathbb{R}}$, the rays being numbered $\sigma_{1}, \sigma_{3}, \ldots$ with odd indices, and the dotted lines represent each of the Equations 1.4. The shaded area is the divisor polytope, and equivalently, up to identifying spaces as in [LM09, Proposition 6.1, (i)], 
the Newton-Okounkov body.
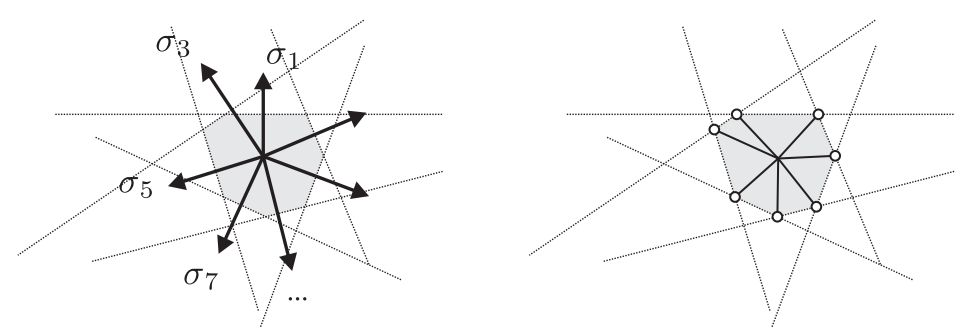

The bullet points above on the right correspond to the functions $h_{\alpha}$. Their convex hull agrees with the divisor polytope. Having the subject of this paper in mind, these bullet points amount to what we want to have on the left side of Equation 0.3 in the introduction.

The idea of the previous example also works for general varieties if the graded semigroup is finitely generated.

Lemma 1.8. We make the same assumptions as in Proposition 1.5. If the graded semigroup $\Gamma_{\underline{v}}(D)$ is finitely generated, then the claim of Proposition 1.5 is true with the additional property that the trivialization polytope is the entire Newton-Okounkov body.

Proof. (Step 1) Let $\left(\underline{v}\left(s_{i}\right), m_{i}\right)$ be a finite set of semigroup generators for $\Gamma_{\underline{v}}(D)$. We claim that we may simplify Equation 1.2 to

$$
\Delta_{\underline{v}}(D)=\text { convex hull }\left\{\frac{1}{m_{i}} \underline{v}\left(s_{i}\right)\right\} .
$$

This holds since any $(\underline{v}(s), m)$ in Equation 1.1 can be written as

$$
(\underline{v}(s), m)=\sum_{i} n_{i}\left(\underline{v}\left(s_{i}\right), m_{i}\right) \quad \text { for } \quad n_{i} \in \mathbb{Z}_{\geq 1} .
$$

The line segment from $(0, \ldots, 0)$ to $(\underline{v}(s), m)$ intersects the $m=1$ hyperplane at

$$
\frac{1}{m}(\underline{v}(s), m)=\frac{1}{\sum_{j} n_{j} m_{j}}\left(\sum_{i} n_{i} \underline{v}\left(s_{i}\right), \sum_{i} n_{i} m_{i}\right)=\left(\sum_{i}\left(\frac{n_{i} \cdot m_{i}}{\sum n_{j} m_{j}}\right) \frac{1}{m_{i}} \underline{v}\left(s_{i}\right), 1\right)
$$

and we have $\frac{n_{i} \cdot m_{i}}{\sum n_{j} m_{j}} \in[0,1]$ and the sum of all these coefficients is 1 , so this is a convex combination of the vectors in Equation 1.5. Being the convex hull of finitely many points, we do not need to take the topological closure in Equation 1.5. We have $\operatorname{div}\left(s_{i}\right) \geq-m_{i} D$ and since the divisor is supported in a closed subscheme of codimension $\geq 1$, we may pick a dense open $U$ such that we have equality $\left.\operatorname{div}\left(s_{i}\right)\right|_{U}=-\left.m D\right|_{U}$. We may add this open to any given finite trivialization; repeat this for all $i$.

(Step 2) Since $\frac{1}{m_{i}} \underline{v}\left(s_{i}\right)=\frac{1}{m_{i} N} \underline{v}\left(s_{i}^{N}\right)$ for any $N \geq 1$, we may pick a common multiple of the denominators, and then Equation 1.5 can be rewritten as $\Delta_{\underline{v}}(D)=$ convex hull $\left\{\frac{1}{N} \underline{v}\left(s_{i}^{\prime}\right)\right\}$ for suitable $s_{i}^{\prime}:=s_{i}^{N / m_{i}} \in H^{0}\left(X, \mathcal{O}_{X}(N \cdot D)\right)$ and $N$ independent of $i$. We have arrived the claim of Proposition 1.5. but now the trivialization polytope agrees with $\Delta_{\underline{v}}(D)$ instead of merely being contained in it.

Example 1.9. Under similar assumptions as in Lemma 1.8, Anderson constructs a family $\tilde{X} \rightarrow \mathbb{A}^{1}$ such that the central fiber $\tilde{X}_{0}$ (up to normalization) agrees with the toric variety of the polytope $\Delta_{Y_{\mathbf{v}}}(D)$, and all other fibers $\tilde{X}_{t}, t \neq 0$, are isomorphic to $X$, And13. Along this degeneration, Lemma 1.8 transforms into the situation discussed in Example 1.7 


\section{The InTERSECTION FORM}

2.1. Intersection form via topology. Let us first recall some basics around intersection numbers. In his book Laz04], Lazarsfeld uses the following elementary approach: Suppose $X / \mathbb{C}$ is an $n$-dimensional integral proper variety. Then we may attach to any line bundle $L$ its first Chern class $c_{1}(L) \in H^{2}(X, \mathbb{Z})$, which can in turn be defined as the image of the connecting map of the exponential sequence of sheaves

$$
0 \longrightarrow \mathbb{Z} \stackrel{\cdot 2 \pi i}{\longrightarrow} \mathcal{O}_{X} \stackrel{\exp }{\longrightarrow} \mathcal{O}_{X}^{\times} \longrightarrow 0
$$

namely

$$
H^{1}\left(X, \mathcal{O}_{X}^{\times}\right) \longrightarrow H^{2}(X, \mathbb{Z})
$$

Now, just using the product in the singular cohomology ring, one obtains the intersection form

$$
\int_{X} L_{1} \cdots L_{n}:=\int_{X} c_{1}\left(L_{1}\right) \smile \cdots \smile c_{1}\left(L_{n}\right) \in \mathbb{Z},
$$

where the integral on the right denotes the evaluation against the fundamental class $[X] \in$ $H_{2 n}(X, \mathbb{Z})$. In other words, the intersection form arises as the composition of

$$
\begin{aligned}
& H^{1}\left(X, \mathcal{O}_{X}^{\times}\right) \otimes \cdots \otimes H^{1}\left(X, \mathcal{O}_{X}^{\times}\right) \\
& \longrightarrow H^{2}(X, \mathbb{Z}) \otimes \cdots \otimes H^{2}(X, \mathbb{Z}) \stackrel{\smile}{\longrightarrow} H^{2 n}(X, \mathbb{Z}) \stackrel{\cdot[X]}{\longrightarrow} \mathbb{Z} .
\end{aligned}
$$

In this text, we shall use a different approach based on Milnor $K$-theory. It is based on writing Chow groups as sheaf cohomology groups:

Theorem 2.1 (Bloch-Quillen, Grayson). Suppose $X / k$ is a smooth variety. Then there is a canonical ring isomorphism

$$
\mathrm{CH}^{*}(X) \cong H_{\mathrm{Zar}}^{*}\left(X, \mathcal{K}_{*}^{\mathrm{M}}\right),
$$

where $\mathcal{K}_{*}^{\mathrm{M}}$ is the Milnor $K$-theory sheaf (whose definition we recall below). On the right-hand side we use sheaf cohomology with respect to the Zariski topology on $X$.

History 2.2. This method is not very well-known, so let us provide some background: In degree one it boils down to $\mathrm{CH}^{1}(X) \cong H_{\mathrm{Zar}}^{*}\left(X, \mathcal{O}_{X}^{\times}\right)$, i.e. the classical isomorphism between Weil divisor classes and Cartier divisor classes. The idea to generalize this to higher codimension is due to Bloch, who discovered that codimension two algebraic cycles are related to $K_{2}$-groups; he even speaks of "codimension two Cartier divisors" in Blo74. Quillen proved the general version. These papers used full algebraic $K$-theory, but Kato later discovered that Milnor $K$-theory also works. Grayson proved that the intersection product also matches the sheaf cup product structure on the right side in the context of algebraic $K$-theory Gra78. In this paper we use a more modern formulation due to Rost [Ros96] using Milnor $K$-theory which has nice signs. See also Gillet Gil05.

Let us carefully explain how to use the above theorem for the computation of intersection numbers. But first we should recall the definition of the sheaves $\mathcal{K}_{*}^{\mathrm{M}}$, which we will do in the next section.

2.2. Milnor $K$-groups. Let $F$ be any field. Then $F^{\times}$is an abelian group, and thus a $\mathbb{Z}$-module. Write

$$
T F^{\times}:=\bigoplus_{p \geq 0} \underbrace{F^{\times} \otimes_{\mathbb{Z}} \cdots \otimes_{\mathbb{Z}} F^{\times}}_{p \text { factors }}
$$


for the free tensor algebra of $F^{\times}$, as a $\mathbb{Z}$-module. The unusal rôle of using powers to define the $\mathbb{Z}$-module structure can be confusing at first. For example, for any integer $n \in \mathbb{Z}$, it means that

$$
n \cdot\left(f_{1} \otimes f_{2}\right)=f_{1}^{n} \otimes f_{2}=f_{1} \otimes f_{2}^{n} \quad f_{1} \otimes \frac{1}{f_{2}}=\frac{1}{f_{1}} \otimes f_{2} .
$$

Now define the Milnor $K$-theory ring as

$$
K_{*}^{\mathrm{M}}(F):=\frac{T F^{\times}}{\langle x \otimes(1-x)| \text { for all } x \in F \backslash\{0,1\}\rangle},
$$

i.e. we quotient out the two-sided ideal generated by all pure tensors $x \otimes(1-x)$ for $x \in$ $F \backslash\{0,1\}$. This is called the Steinberg relation. It is customary to write

$$
\left\{f_{1}, \ldots, f_{p}\right\}:=f_{1} \otimes \cdots \otimes f_{p}
$$

for the elements in this quotient ring. We obtain that $K_{*}^{\mathrm{M}}(F)$ is a $\mathbb{Z}$-graded ring. In degrees 0 and 1 it is easy to understand

$$
K_{*}^{\mathrm{M}}(F)=\mathbb{Z} \oplus F^{\times} \oplus K_{2}^{\mathrm{M}}(F) \oplus K_{3}^{\mathrm{M}}(F) \oplus \ldots
$$

In general $K_{p}^{\mathrm{M}}(F)$ for $p \geq 2$ is rather uncomputable except for very special cases. However, we will never really need to know what these groups are, so this is harmless.

Lemma 2.3 ([Mil70, Lemma 1.1 and 1.2]). $K_{*}^{\mathrm{M}}(F)$ is graded-commutative, and moreover $\{x, x\}=\{x,-1\}$ holds for any $x \in F^{\times}$.

Lemma 2.4. Let $F$ be a field and $v$ a discrete rank one valuation. Then every element in $K_{p}^{\mathrm{M}}(F)$ is a finite $\mathbb{Z}$-linear combination of elements of the shapes

$$
\left\{\pi, u_{2}, \ldots, u_{p}\right\} \quad \text { and } \quad\left\{u_{1}, u_{2}, \ldots, u_{p}\right\},
$$

where $\pi$ is a uniformizer, i.e. $v(\pi)=1$, and $u_{1}, \ldots, u_{p} \in \mathcal{O}_{v}^{\times}$have valuation zero.

Proof. It suffices to prove this for a pure tensor $\left\{f_{1}, \ldots, f_{p}\right\}$. Every element $f \in F^{\times}$can be written as $u \pi^{k}$ for $u \in \mathcal{O}_{v}^{\times}, k \in \mathbb{Z}$. Do this in every slot. Then multi-linearity and the relation $\{\pi, \pi\}=\{\pi,-1\}$ (Lemma 2.3) yield the claim.

We need a few more constructions.

C1 (Restriction) If $L / F$ is an arbitrary field extension, there is a natural graded ring homomorphism

$$
\operatorname{res}_{L / F}: K_{*}^{\mathrm{M}}(F) \longrightarrow K_{*}^{\mathrm{M}}(L)
$$

simply by using the inclusion $F^{\times} \hookrightarrow L^{\times}$slot by slot.

C2 (Norms $\bigcap^{1}$ If $L / F$ is a finite field extension, there is a natural map

$$
N_{L / K}: K_{p}^{\mathrm{M}}(L) \longrightarrow K_{p}^{\mathrm{M}}(F)
$$

in each degree $p$. It does not preserve the ring structure. In degree 0 it is $\mathbb{Z} \stackrel{\cdot[L: F]}{\longrightarrow} \mathbb{Z}$, and in degree 1 it is the usual norm map $L^{\times} \longrightarrow F^{\times}$. The construction of this map in higher degrees is complicated. We refer to [GS06, §7.3]. The norm is functorial in towers, i.e. if

$$
F \subseteq L \subseteq L^{\prime}
$$

are two finite field extensions,

$$
N_{L^{\prime} / F}=N_{L / F} \circ N_{L^{\prime} / L}
$$

\footnotetext{
${ }^{1}$ This is called 'corestriction' or 'norm' in the literature, depending on the taste of authors.
} 
C3 (Boundary map) If $v$ is a discrete rank one valuation on $F$, let $\mathcal{O}_{v} \subset F$ denote its valuation ring with maximal ideal $\mathfrak{m}_{v}$. Write $\kappa(v):=\mathcal{O}_{v} / \mathfrak{m}_{v}$ for its residue field. There is a natural map

$$
\partial_{v}: K_{p}^{\mathrm{M}}(F) \longrightarrow K_{p-1}^{\mathrm{M}}(\kappa(v)) .
$$

In degree 1 it is $F^{\times} \longrightarrow \mathbb{Z}, f \mapsto v(f)$, the valuation itself. For $p \geq 2$, it can fully be described as follows: If $\pi$ is a uniformizer for $v$, i.e. $v(\pi)=1$, and $u_{1}, \ldots, u_{p} \in \mathcal{O}_{v}^{\times}$then define

$$
\begin{aligned}
\partial_{v}\left\{\pi, u_{2}, \ldots, u_{p}\right\} & :=\left\{\overline{u_{2}}, \ldots, \overline{u_{p}}\right\} \\
\partial_{v}\left\{u_{1}, u_{2}, \ldots, u_{p}\right\} & :=0 .
\end{aligned}
$$

and by Lemma 2.4 this suffices to uniquely pin down the map. We neglect proving here that this amounts to a well-defined map. The map is independent of the choice of the uniformizer $\pi$.

There are various relations between the maps of C1-C3. We shall only need very few. Most importantly, we will use the compatibility between boundary maps and norms.

Proposition 2.5 (GS06, Ch. 7, Corollary 7.4.3]). Suppose L is a discrete valuation field with valuation $v$ and residue field $\kappa(v)$. Write $\mathcal{O}_{v}$ for its valuation ring. Let $L^{\prime} / L$ be a finite field extension. Write $\mathcal{O}_{v}^{\prime}$ for the integral closure of the valuation ring $\mathcal{O}_{v}$ inside $L^{\prime}$. Suppose $\mathcal{O}_{v}^{\prime}$ is a finite $\mathcal{O}_{v}$-module (this will hold whenever we have finiteness of integral closures, e.g. if $\mathcal{O}_{v}$ is a valuation ring coming from a variety). Then $\mathcal{O}_{v}^{\prime}$ is a semi-local ring and if $w$ runs through the discrete valuations extending $v$, the diagram

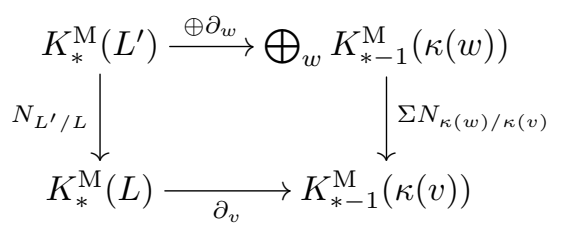

commutes.

2.3. The boundary map between points. We need to recall a refinement of the boundary map $\partial_{v}$ for schemes. The main point is that we wish to attach a boundary map to any codimension one subscheme, but outside the normal locus, a codimension one scheme does not uniquely pin down a discrete rank one valuation. The construction is a little involved. The reader may prefer to skip it initially, keeping in mind that 'generically' Remark 2.7 can be used instead.

Let $Y / k$ be an integral variety. Suppose $Z \hookrightarrow Y$ is an integral closed subscheme of codimension one. The underlying closed subset of $Z$ is irreducible and thus has a unique generic point, call it $z$. Being of codimension one, the local ring $\mathcal{O}_{Y, z}$ is a one-dimensional local domain. It is contained in the rational function field $k(Y)$ (the local ring at the generic point of $Y$ itself). Its residue field $\kappa(z)$ agrees with the rational function field $k(Z)$ of $Z$.

Let $\mathcal{O}_{Y, z}^{\prime}$ denote the integral closure of $\mathcal{O}_{Y, z}$ inside $k(Y)$, i.e.

$$
\operatorname{Spec} \mathcal{O}_{Y, z}^{\prime} \longrightarrow \operatorname{Spec} \mathcal{O}_{Y, z}
$$

is the normalization morphism. This is a finite morphism of schemes since $Y$ was assumed to be of finite type over a field (finiteness of integral closure). Thus, the $\operatorname{ring} \mathcal{O}_{Y, z}^{\prime}$ is semi-local. Let $\mathfrak{m}_{1}, \ldots, \mathfrak{m}_{r}$ denote its maximal ideals. Then each localization $\left(\mathcal{O}_{Y, z}^{\prime}\right)_{\mathfrak{m}_{i}} \subset k(Y)$ is a onedimensional normal domain and thus a discrete valuation ring. Let $v_{i}$ be the discrete rank one valuation of $k(Y)$ coming from $\left(\mathcal{O}_{Y, z}^{\prime}\right)_{\mathfrak{m}_{i}}$. The residue field of the valuation $v_{i}$ agrees with the residue field of $\left(\mathcal{O}_{Y, z}^{\prime}\right)_{\mathfrak{m}_{i}}$, so we call it $\kappa\left(v_{i}\right)$. Since each $\mathfrak{m}_{i}$ under the finite morphism of 
Equation 2.10 maps to $\mathfrak{m}_{z}$, we get attached residue field extensions $\kappa\left(v_{i}\right) / \kappa(z)$ and these are themselves finite.

Definition 2.6. Let $Y / k$ be an integral variety. Suppose $Z \hookrightarrow Y$ is an integral closed subscheme of codimension one. Define

$$
\partial_{Z}^{Y}: K_{p}^{\mathrm{M}}(k(Y)) \stackrel{\oplus \partial_{v_{i}}}{\longrightarrow} \bigoplus_{i=1}^{r} K_{p-1}^{\mathrm{M}}\left(\kappa\left(v_{i}\right)\right) \stackrel{\oplus N_{\kappa\left(v_{i}\right) / k(Z)}}{\longrightarrow} K_{p-1}^{\mathrm{M}}(k(Z)),
$$

where the first arrow stems from the boundary maps $\partial_{v_{i}}$ of Equation 2.6, and each $N_{(-) /(-)}$ is a norm map as in Equation 2.4.

Remark 2.7. If $\mathcal{O}_{Y, z}$ is normal, it is itself a discrete valuation ring. Write $v$ for its valuation. In this special case the above definition simplifies to $\partial_{Z}^{Y}=\partial_{v}$, i.e. we are back in the situation of Equation 2.6.

2.4. The Milnor $K$-sheaf. Let $X / k$ be a variety.

Definition 2.8. For any Zariski open $U \subseteq X$ define

$$
\mathcal{K}_{p}^{\mathrm{M}}(U):=\operatorname{ker}\left(\bigoplus_{Y_{0}} K_{p}^{\mathrm{M}}\left(k\left(Y_{0}\right)\right) \stackrel{\partial_{Y_{1}}^{Y_{0}}}{\longrightarrow} \bigoplus_{Y_{1}} K_{p-1}^{\mathrm{M}}\left(k\left(Y_{1}\right)\right)\right),
$$

where $Y_{i} \subseteq U$ runs through all integral closed subschemes of codimension $i$ (for $\left.i=0,1\right)$. The map $\partial_{Y_{1}}^{Y_{0}}$ was set up in Definition 2.6.

Note that if $X$ is integral, this simplifies to

$$
\operatorname{ker}\left(K_{p}^{\mathrm{M}}(k(X)) \stackrel{\partial_{Y_{1}}^{X}}{\longrightarrow} \bigoplus_{Y_{1}} K_{p-1}^{\mathrm{M}}\left(k\left(Y_{1}\right)\right)\right) \subseteq K_{p}^{\mathrm{M}}(k(X)),
$$

so if we go to a smaller open $U^{\prime} \subseteq U$, we at worst remove conditions when forming the kernel, so it is clear that $\mathcal{K}_{p}^{\mathrm{M}}(-)$ defines a presheaf. Argueing individually for each irreducible component, this works in general without requiring $X$ to be integral. One can further check that $\mathcal{K}_{p}^{\mathrm{M}}$ defines a sheaf in the Zariski topology. There is a product structure

$$
\smile: \mathcal{K}_{p}^{\mathrm{M}} \otimes \mathcal{K}_{q}^{\mathrm{M}} \longrightarrow \mathcal{K}_{p+q}^{\mathrm{M}}
$$

making $\mathcal{K}_{*}^{\mathrm{M}}:=\bigoplus_{p>0} \mathcal{K}_{p}^{\mathrm{M}}$ a Zariski sheaf of graded commutative algebras. The product is defined simply by concatenation (and thus compatible to the one induced from the tensor algebra in the definition for fields, Equation 2.3,

$$
\left\{x_{1}, \ldots, x_{p}\right\} \smile\left\{y_{1}, \ldots, y_{q}\right\}:=\left\{x_{1}, \ldots, x_{p}, y_{1}, \ldots, y_{q}\right\},
$$

but one must check that this still lies in the kernel as in Equation 2.11. This works as follows.

If $v$ is a discrete rank one valuation on a field $F$ and $\pi \in \mathcal{O}_{v}$ a chosen uniformizer, one can define the specialization map

$$
s_{v}^{\pi}: K_{p}^{\mathrm{M}}(F) \longrightarrow K_{p}^{\mathrm{M}}(\kappa(v)), \quad\left\{x_{1}, \ldots, x_{p}\right\} \longmapsto \partial_{v}\left\{-\pi, x_{1}, \ldots, x_{p}\right\} .
$$

Unlike $\partial_{v}$, these maps depend on the uniformizer $\pi$. Based on this, there is an extended "non-linear" Leibniz formula for the maps $\partial_{v}$ : For $x \in K_{p}^{\mathrm{M}}(F)$ and $y \in K_{q}^{\mathrm{M}}(F)$, we have

$$
\partial_{v}(x \cdot y)=\partial_{v}(x) \cdot s_{v}^{\pi}(y)+(-1)^{p} s_{v}^{\pi}(x) \cdot \partial_{v}(y)+\{-1\} \cdot \partial_{v}(x) \cdot \partial_{v}(y)
$$

valid irrespective of the choice of the uniformizer. Thus, if $\partial_{v}(x)=0$ and $\partial_{v}(y)=0$, it follows that $\partial_{v}(x \cdot y)=0$, giving Equation 2.12 .

Lemma 2.9. Suppose $X$ is normal. There is an isomorphism of sheaves 
(1) $\mathcal{K}_{0}^{\mathrm{M}} \cong \mathbb{Z}$

(2) $\mathcal{K}_{1}^{\mathrm{M}} \cong \mathcal{O}_{X}^{\times}$.

Proof. (1) follows since $K_{0}^{\mathrm{M}}(F)=\mathbb{Z}$ and $K_{-1}^{\mathrm{M}}(F)=0$ for any field $F$. The direct sum over $Y_{0}$ in Equation 2.11 is exactly the same which would arise from Zariski sheafification of the constant presheaf $\mathbb{Z}$. (2) We find

$$
\mathcal{K}_{1}^{\mathrm{M}}(U):=\operatorname{ker}\left(\bigoplus_{Y_{0}} k\left(Y_{0}\right)^{\times} \stackrel{\partial_{v}}{\longrightarrow} \bigoplus_{Y_{1}} \mathbb{Z}\right),
$$

where $v$ runs through all discrete rank one valuations of the function field $k\left(Y_{0}\right)$. For a normal integral scheme, we have equality of rings

$$
\mathcal{O}_{X}(U)=\bigcap_{v} \mathcal{O}_{v} \subseteq k\left(Y_{0}\right),
$$

where $v$ runs through all rank one valuations. In particular, units in $\mathcal{O}_{X}(U)^{\times}$are units in all $\mathcal{O}_{v}$, and this amounts to $\partial_{v}(x)=0$ for all $v$.

One can widely generalize Lemma 2.9 to all $\mathcal{K}_{n}^{\mathrm{M}}$, Ker09. While aesthetically very pleasant, this is not of any use for our immediate purposes.

2.5. Intersection form - reprise. We may now explain how to rephrase the intersection form. Recall from 2.1 that Lazarsfeld uses the intersection form

$$
\begin{aligned}
H^{1}\left(X, \mathcal{O}_{X}^{\times}\right) & \otimes \cdots \otimes H^{1}\left(X, \mathcal{O}_{X}^{\times}\right) \\
& \longrightarrow H^{2}(X, \mathbb{Z}) \otimes \cdots \otimes H^{2}(X, \mathbb{Z}) \stackrel{\smile}{\longrightarrow} H^{2 n}(X, \mathbb{Z}) \stackrel{\cdot[X]}{\longrightarrow} \mathbb{Z} .
\end{aligned}
$$

on a complex variety. Here the first arrow stems from the first Chern class map $c_{1}$ : $H^{1}\left(X, \mathcal{O}_{X}^{\times}\right) \rightarrow H^{2}(X, \mathbb{Z})$ from the exponential sequence, Equation 2.1 applied to each tensor slot individually. On the derived level, we can write the latter as a map

$$
\mathcal{O}_{X}^{\times} \stackrel{c_{1}}{\longrightarrow} \mathbb{Z}[1]
$$

where $\mathbb{Z}[1]$ is a complex concentrated in degree one.

Remark 2.10. More precisely, the exact sequence in Equation 2.1 defines an extension class in the group $\operatorname{Ext}^{1}\left(\mathcal{O}_{X}^{\times}, \mathbb{Z}\right)$ in the category of sheaves with values in abelian groups. In the derived category this Ext-group gets interpreted as $\operatorname{Ext}^{m}\left(\mathcal{O}_{X}^{\times}, \mathbb{Z}\right)=\mathbf{R} \operatorname{Hom}\left(\mathcal{O}_{X}^{\times}, \mathbb{Z}[m]\right)$, giving Equation 2.15.

Note that under the tensor product of complexes, this means that $\mathbb{Z}[p] \otimes \mathbb{Z}[q] \cong \mathbb{Z}[p+q]$ (which one sees by taking the total complex of the tensor product complex). Thus, from this angle, the first arrow in Equation 2.14 really stems from

$$
\mathcal{O}_{X}^{\times} \otimes \cdots \otimes \mathcal{O}_{X}^{\times} \longrightarrow \mathbb{Z}[1] \otimes \cdots \otimes \mathbb{Z}[1] \stackrel{\sim}{\longrightarrow} \mathbb{Z}[n]
$$

and in cohomology this induces a map

$$
H^{i}\left(X, \mathcal{O}_{X}^{\times} \otimes \cdots \otimes \mathcal{O}_{X}^{\times}\right) \longrightarrow H^{i}(X, \mathbb{Z}[n]) \stackrel{\sim}{\longrightarrow} H^{i+n}(X, \mathbb{Z}) .
$$


Thus, we observe that the construction used in Lazarsfeld's book Laz04 factors over the top row in the diagram

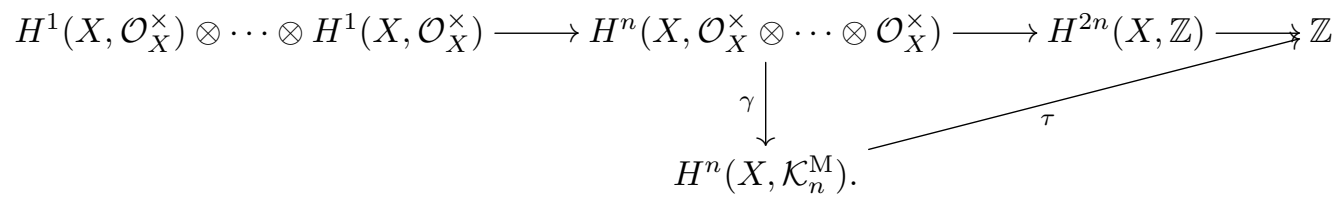

The map $\gamma$ is induced from the isomorphism of sheaves $\mathcal{K}_{1}^{\mathrm{M}} \cong \mathcal{O}_{X}^{\times}$(see Lemma 2.9 and then uses the graded-commutative product structure of the sheaf $\mathcal{K}_{p}^{\mathrm{M}} \otimes \mathcal{K}_{q}^{\mathrm{M}} \longrightarrow \mathcal{K}_{p+q}^{\mathrm{M}}$ to get to $\mathcal{K}_{n}^{\mathrm{M}}$. Note that this map $\gamma$ replaces the use of the first Chern class in Equation 2.16 .

What is not at all obvious, is that the factorization $\tau$ exists, and this is where Theorem 2.1 enters. However, for our purposes we only really need an explicit formula for $\tau$ :

Theorem 2.11. Let $X / k$ be an $n$-dimensional irreducible smooth proper variety. Let $\mathfrak{U}=$ $\left(U_{i}\right)_{i \in I}$ be an open cover where $I$ is some index set. Suppose

$$
X=\bigcup_{\alpha \in I} \Sigma_{\alpha} \quad \text { with } \quad \Sigma_{\alpha} \subseteq U_{\alpha}
$$

is a disjoint decomposition as a set. For $p \in\{1, \ldots, n\}$ let $L_{p}$ be a line bundle on $X$, and suppose it can locally be trivialized on the open cover $\mathfrak{U}$, so that its 1-cocycle takes the form

$$
\left(f_{\alpha, \beta}^{p}\right)_{\alpha, \beta \in I} \in \check{H}^{1}\left(\mathfrak{U}, \mathcal{O}_{X}^{\times}\right)
$$

in Čech cohomology. Then

$\int_{X} c_{1}\left(L_{1}\right) \cdots c_{1}\left(L_{n}\right)=\sum_{Y_{\bullet}}\left[k\left(Y_{n}\right): k\right] \partial_{Y_{n}}^{Y_{n-1}} \cdots \partial_{Y_{1}}^{Y_{0}}\left\{f_{\alpha\left(Y_{0}\right) \alpha\left(Y_{1}\right)}^{1}, f_{\alpha\left(Y_{1}\right) \alpha\left(Y_{2}\right)}^{2}, \ldots, f_{\alpha\left(Y_{n-1}\right) \alpha\left(Y_{n}\right)}^{n}\right\}$, where

(1) the sum runs over all flags

$$
Y_{\bullet}: Y_{n} \subset \cdots \subset Y_{1} \subset Y_{0}
$$

in the sense of Definition 1.1 (i.e. they need not be admissible!);

(2) for any integral closed subscheme $Y \hookrightarrow X$ let $\alpha(Y)$ denote the unique index $\alpha$ such that the generic point of $Y$ lies in $\Sigma_{\alpha}$ (see Equation 2.18);

(3) $k\left(Y_{n}\right)$ denotes the function field of the closed point $Y_{n}$ (i.e. it is the residue field of this scheme point).

On the right-hand side there exist only finitely many flags $Y_{\bullet}$ such that the summand is non-zero.

This is [Bra13, Proposition 3]. See the rest of the cited paper for more background on how this formula for intersection multiplicities works.

Remark 2.12. A few remarks on the intersection theory being used: If $D_{1}, \ldots, D_{n}$ are divisors such that $\bigcap D_{i}=p t$ is a single closed point, the local intersection index can be computed via

$$
\operatorname{res}_{p t} \frac{\mathrm{d} f_{1}}{f_{1}} \wedge \cdots \wedge \frac{\mathrm{d} f_{n}}{f_{n}}
$$

where $f_{i}$ is a local equation for $D_{i}$ and $\operatorname{res}_{p t}$ is a somewhat shortened abbreviation for Grothendieck's residue symbol, see Griffiths-Harris [GH94, p. 663, and end of page 669]. Any such expression can be rephrased in terms of rank $n$ valuation vectors, by a variant of 
Proposition 3.9 below for residues. However, for ample divisors, the volume is related to the top self-intersection number, so all $D_{i}$ agree, and the condition $\bigcap D_{i}=p t$ fails miserably (and even if it did not, the $n$-form in Equation 2.19 is zero). One could fix this issue by moving each $D_{i}$ within its linear equivalence class, rather non-canonically. However, the formulation of intersection numbers as above does not run into this problem at all. It would turn out to express intersection numbers in terms of rank $n$ valuation vectors, irrespective of the dimension of the set-theoretic intersection, in fact this would even work if the self-intersection was highly negative.

\section{THE MAIN THEOREM}

\subsection{Top rank valuations and $n$-DVFs.}

Definition 3.1. We define an $n$-DVF with last residue field $\kappa$ (short for " $n$-discrete valuation field") as follows:

- A 0-DVF with last residue field $\kappa$ is the field $\kappa$ itself.

- An $n$-DVF with last residue field $\kappa$ is a field $(F, v)$ with a discrete rank one valuation $v$ such that $\kappa(v)$ is an $(n-1)-D V F$ with last residue field $\kappa$.

Although it is cleanest to use this inductive definition, one may visualize an $n$-DVF $F$ as the following structure

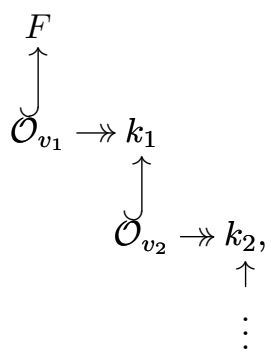

where each $\mathcal{O}_{v_{i}}$ is the valuation ring for the discrete rank one valuation $v_{i}$ defined on the field displayed above $\mathcal{O}_{v_{i}}$ (so that the upward arrows are the inclusions of the valuation rings), while each surjection to the right is the quotient map $\mathcal{O}_{v_{i}} \rightarrow \mathcal{O}_{v_{i}} / \mathfrak{m}_{v_{i}}=: k_{i}$ to the respective residue field.

Suppose $X / k$ is an irreducible $n$-dimensional smooth projective variety. Let $k(X)$ be its function field. If $\underline{v}: k(X)^{\times} \rightarrow \mathbb{Z}_{\text {lex }}^{n}$ is a top rank valuation, it takes values in $\mathbb{Z}_{\text {lex }}^{n}$, lexicographically ordered, and one can write it as $\underline{v}(f)=\left(\underline{v}_{1}(f), \ldots, \underline{v}_{n}(f)\right)$, where $\underline{v}_{i}(f)$ : $k(X)^{\times} \rightarrow \mathbb{Z}$ is our notation for the components of this valuation vector.

Remark 3.2. This construction would make sense for any field instead of " $k(X)$ ", but we will only use it in the said context.

It turns out that $\underline{v}_{1}$ is a rank one $\mathbb{Z}$-valued valuation on $k(X)^{\times}$, defining a valuation ring $\mathcal{O}_{v_{1}}$; and this can be continued interatively along zig-zag as in Figure 3.1. So, every top rank valuation $\underline{v}: k(X)^{\times} \longrightarrow \mathbb{Z}_{\text {lex }}^{n}$ determines the structure of an $n$-DVF with last residue field being the residue field of $\underline{v}$.

Example 3.3. Every admissible flag in the sense of Definition 1.1 defines a top rank valuation. If we allow ourselves to switch to a different birational model, any finite number of top rank valuations can be made to come from admissible flags, [CFK ${ }^{+} 17$, Theorem 2.9]. 
Conversely, giving the structure of an $n$-DVF on $k(X)$, then if we addtionally pick uniformizers, it pins down a unique valuation $\underline{v}: k(X)^{\times} \longrightarrow \mathbb{Z}_{\text {lex }}^{n}$ :

Definition 3.4. Let $F$ be an $n-D V F$. Fix uniformizers $\pi_{i} \in \mathcal{O}_{v_{i}}$. Define a rank $n$ valuation

$$
\begin{aligned}
& \underline{v}: F^{\times} \longrightarrow \mathbb{Z}_{\operatorname{lex}}^{n}
\end{aligned}
$$

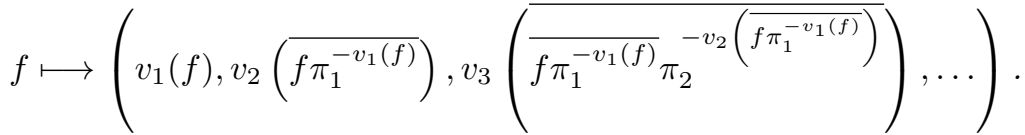

For $n \geq 2$ this valuation depends on the choice of the uniformizers.

We have intentionally mimicked the definitions in [LM09, §1.1] to stress the analogy.

Remark 3.5. Let us explain the idea and also give a more precise definition: The first entry is just the valuation $v_{1}$ itself. Next, note that $f \pi_{1}^{-v_{1}(f)}$ by construction has valuation zero, so it lies in $\mathcal{O}_{v_{1}}^{\times}$and thus its image under the quotient map to the residue field is also invertible, i.e.

$$
\overline{f \pi_{1}^{-v_{1}(f)}} \in k_{1}^{\times} .
$$

Hence, it makes sense to consider its valuation with respect to $v_{2}$. Now repeat this. That is, we multiply with an appropriate power of $\pi_{2}$ to ensure we get an element in $\mathcal{O}_{v_{2}}^{\times}$. It is more elegant to define the higher rank valuation by inductively defining

$$
f_{1}:=f \quad \text { and } \quad f_{p+1}:=\overline{f_{p} \pi_{p}^{-v_{p}\left(f_{p}\right)}}
$$

for $p \geq 1$ and $f_{p+1} \in k_{p}$. Then

$$
f \mapsto\left(v_{1}\left(f_{1}\right), v_{2}\left(f_{2}\right), \ldots, v_{n}\left(f_{n}\right)\right) .
$$

Unravelling the inductive nature of this definition, we find Equation 3.2

Example 3.6. Consider $X:=\mathbb{A}_{k}^{2}=\operatorname{Spec} k[s, t]$. It has a 2-DVF structure coming from the flag of ideals $(s, t) \subset(t) \subset(0)$,

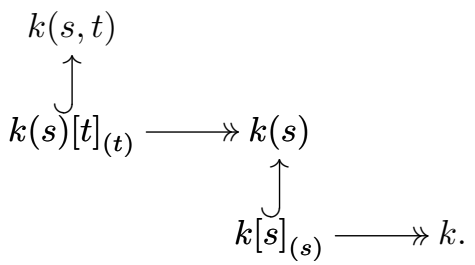

Then for any $m \in \mathbb{Z}, \pi_{1}:=s^{-m} t$ is a uniformizer for $k(s)[t]_{(t)}$. We obtain the valuation vector $\underline{v}(t)=(1, m)$, depending on $m$. This cannot happen for the valuation vectors in the definition of Newton-Okounkov bodies. The whole point is that $\pi_{1}:=s^{-m} t$ only lies in the local ring $k[s, t]_{(s, t)}$ of the variety if $m \leq 0$, and then is only a local equation for $(t)$ if $m=0$, for otherwise it is a local equation for a two component subvariety, with components cut out by $(t)$ and a nil-thickening of $(s)$. Thus, only $m=0$ is possible in the setting of Okounkov bodies. For a general $n$-DVF, there is no analogue of the local ring which allows us to single out such problematic choices of uniformizers.

Let us point out that one can define the valuation vectors which appear in the construction of Newton-Okounkov bodies entirely in the language of Milnor $K$-groups: 
Lemma 3.7. Let $X / k$ be an irreducible smooth proper variety of dimension $n$ and $\underline{v}$ the top rank valuation coming from an admissible flag $Y_{\bullet}$ in $X$. Let $f \in k(X)^{\times}$be given. Let $\pi_{i}$ be the local equations of the individual flag members. Define a vector $\underline{x} \in \mathbb{Z}^{n}$ with components

$$
\underline{x}_{p}:=\partial_{v_{p}} \circ \cdots \circ \partial_{v_{1}}\left\{\pi_{1}, \ldots, \pi_{p-1}, f\right\} .
$$

Then $\underline{v}(f):=\left(\underline{x}_{1}, \ldots, \underline{x}_{n}\right)$.

Note that our choice of the $\pi_{i}$ is more strict that just requiring them to be arbitrary uniformizers in $\mathcal{O}_{v_{i}}$; they all lie in the local ring $\mathcal{O}_{X, Y_{n}}$. One could rewrite the vector as

$$
\underline{x}_{1}=\partial_{v_{1}}\{f\} \quad \underline{x}_{p}:=\partial_{v_{p}} s_{v_{p-1}}^{-\pi_{p-1}} \cdots s_{v_{1}}^{-\pi_{1}}\{f\}
$$

for $p \geq 2$ using the specialization maps of Equation 2.13 .

Proof. Since all valuation vector components lie in $\mathbb{Z}$, we can throughout the proof neglect elements which are 2-torsion, for once we map our constructs to $\mathbb{Z}, 2$-torsion elements must necessarily go to zero. Write $f=u_{m} \pi_{m}^{v_{m}(f)}$ and then for any $m$ such that $1 \leq m \leq p$, we compute

$$
\begin{aligned}
\partial_{v_{p}} & \cdots \circ \partial_{v_{m}}\left\{\pi_{m}, \ldots, \pi_{p-1}, f\right\} \\
& =\partial_{v_{p}} \circ \cdots \circ \partial_{v_{m}}\left\{\pi_{m}, \ldots, \pi_{p-1}, u_{m}\right\}+v_{m}(f) \partial_{v_{p}} \circ \cdots \circ \partial_{v_{m}}\left\{\pi_{m}, \ldots, \pi_{p-1}, \pi_{m}\right\} \\
& =\partial_{v_{p}} \circ \cdots \circ \partial_{v_{m+1}}\left\{\overline{\pi_{m+1}}, \ldots, \overline{\pi_{p-1}}, \overline{f \pi_{m}^{-v_{m}(f)}}\right\}+(2 \text {-torsion })
\end{aligned}
$$

by using multi-linearity, Lemma 2.3 and Equation 2.7. For using the latter, we have used that since the $\pi_{i}$ are elements in the local equations for the flag in the local ring of $X$ at $Y_{0}$, their image is a uniformizer in $\mathcal{O}_{v_{i}}$, but necessarily a unit in all $\mathcal{O}_{v_{j}}$ with $j \neq i$. Now we observe that the above equation admits an induction along the variable $m$. Starting with $m=1$, the last slot " $\overline{f \pi_{m}^{-v_{m}(f)}}$ " in Equation 3.5 follows the same inductive description along $m$ as in Equation 3.3 Since both the induction loc. cit. as well as here begin with $f$, it follows that once $m$ equals $p$, we will have arrived at

$$
\underline{x}_{p}={ }_{\text {def }} \partial_{v_{p}} \circ \cdots \circ \partial_{v_{1}}\left\{\pi_{1}, \ldots, \pi_{p-1}, f\right\}=\partial_{v_{p}}\left\{f_{p}\right\}+(2 \text {-torsion }),
$$

with the same meaning of $f_{p}$ as in Equation 3.3 This is just $v_{p}\left(f_{p}\right)$, so we obtain that the vector $\underline{x}$ agrees with the one in Equation 3.4 . This proves our claim.

Note that this gives a reformulation of the definition of Okounkov bodies which does not involve rank $\geq 2$ valuations anymore. Of course, apart from that, it is the same as the usual one.

Definition 3.8 (Milnor- $K$-style Okounkov bodies). Let $X / k$ be an irreducible smooth proper variety of dimension $n$ and $\underline{v}$ the top rank valuation coming from an admissible flag $Y \bullet$ in $X$. Then the Newton-Okounkov body can be rephrased "for Milnor K-theory aficionados" as the closed convex hull of the vectors

$$
\left\{\frac{1}{m}\left(\partial_{v_{1}}\{f\}, \ldots, \partial_{v_{n}} \circ \cdots \circ \partial_{v_{1}}\left\{\pi_{1}, \ldots, \pi_{n-1}, f\right\}\right)\right\}_{f, m}
$$

where $(f, m)$ runs through all pairs $m \geq 1$ and $f \in H^{0}\left(X, \mathcal{O}_{X}(m D)\right) \backslash\{0\}$. The $\pi_{i}$ are arbitrary local equations of the flag entries and $v_{i}$ the rank one valuations arising from $\underline{v}$. 
3.2. Local computations. The following is surely well-known to experts, but since it is quite important for our results, we provide full details. The same type of determinant evaluation is used in [Par83, §2.2].

Proposition 3.9. Let $F$ be an $n$-DVF. Fix uniformizers $\pi_{i} \in \mathcal{O}_{v_{i}}$. Suppose $f_{1}, \ldots, f_{n} \in F^{\times}$. Then

$$
\left(\partial_{v_{n}} \cdots \partial_{v_{1}}\right)\left\{f_{1}, \ldots, f_{n}\right\}=\operatorname{det}\left(\begin{array}{cccc}
\underline{v}_{1}\left(f_{1}\right) & \underline{v}_{2}\left(f_{1}\right) & \cdots & \underline{v}_{n}\left(f_{1}\right) \\
\underline{v}_{1}\left(f_{2}\right) & \underline{v}_{2}\left(f_{2}\right) & & \vdots \\
\vdots & & \ddots & \\
\underline{v}_{1}\left(f_{n}\right) & \cdots & & \underline{v}_{n}\left(f_{n}\right)
\end{array}\right)
$$

where $\underline{v}(f)=\left(\underline{v}_{1}(f), \ldots, \underline{v}_{n}(f)\right)$ encodes the components of the rank $n$ valuation attached to $F$ via Definition 3.4. On the left-hand side the $v_{1}, \ldots, v_{n}$ denote the discrete rank one valuations of the $n$-DVF structure attached to $\underline{v}$, i.e. the valuations which appear in Figure 3.1.

Proof. We prove this by induction on $n$. For $n=1$ the claim just reads

$$
\partial_{v_{1}}\left\{f_{1}\right\}=\underline{v}_{1}\left(f_{1}\right)=v_{1}\left(f_{1}\right)
$$

and thus reduces to the explanation below Equation 2.6. Suppose the proposition is proven for all $\ell$-DVFs with $\ell<n$. Then we prove the case $n$ : (Step 1) Both the left-hand side as well as the right-hand side in Equation 3.6 change sign under swapping $f_{i}$ with $f_{j}$ : On the left side, this is the graded-commutativity of Milnor $K$-theory, i.e. Lemma 2.3. On the right side it amounts to swapping two rows, so it is a standard property of the determinant. Furthermore, both the left and right side are multiplicative in each slot: On the left side, this is already true for the tensor algebra $T F^{\times}$. On the right side, this follows from the multiplicativity of valuations. Next, we claim that both sides vanish if two slots agree, i.e. $f_{i}=f_{j}$ for some $i \neq j$. This is clear: Under anti-commutativity under swapping the two, it follows that both sides must be 2 -torsion. However, both sides take values in $\mathbb{Z}$. Next, we claim that it suffices to show that left and right side agree on all elements of the shape

$$
\text { (A) }\left\{u_{1}, u_{2}, \ldots, u_{n}\right\} \quad \text { and } \quad \text { (B) }\left\{\pi, u_{2}, \ldots, u_{n}\right\} \text {, }
$$

where $\pi$ is a uniformizer for $\mathcal{O}_{v_{1}}$, i.e. $v_{1}(\pi)=1$, and $u_{1}, \ldots, u_{n} \in \mathcal{O}_{v_{1}}^{\times}$. To see this, use exactly the same argument as in the proof of Lemma 2.4. just replace the relation $\{\pi, \pi\}=\{\pi,-1\}$ by using that once two slots agree, both sides are already zero.

(Step 2) For the elements of shape (A) we find $\partial_{v_{1}}\left\{u_{1}, \ldots, u_{n}\right\}=0$ by Equation 2.8. On the other hand, in the matrix on the right side in Equation 3.6 the first colum reads

$$
\left(\begin{array}{c}
\underline{v}_{1}\left(u_{1}\right) \\
\underline{v}_{1}\left(u_{2}\right) \\
\vdots \\
\underline{v}_{1}\left(u_{n}\right)
\end{array}\right)=\left(\begin{array}{c}
0 \\
0 \\
\vdots \\
0
\end{array}\right)
$$

so the determinant also vanishes. We find $\partial_{v_{1}}\left\{\pi, u_{2}, \ldots, u_{n}\right\}=\left\{\overline{u_{2}}, \ldots, \overline{u_{n}}\right\}$ for elements of shape (B) by Equation 2.7. On the other hand, we claim that

$$
\left(\begin{array}{cccc}
\underline{v}_{1}(\pi) & \underline{v}_{2}(\pi) & \cdots & \underline{v}_{n}(\pi) \\
\underline{v}_{1}\left(u_{2}\right) & \underline{v}_{2}\left(u_{2}\right) & & \underline{v}_{n}\left(u_{2}\right) \\
\vdots & & \ddots & \vdots \\
\underline{v}_{1}\left(u_{n}\right) & \cdots & & \underline{v}_{n}\left(u_{n}\right)
\end{array}\right)=\left(\begin{array}{cccc}
1 & 0 & \cdots & 0 \\
0 & \underline{v}_{2}\left(u_{2}\right) & & \underline{v}_{n}\left(u_{2}\right) \\
\vdots & & \ddots & \vdots \\
0 & \underline{v}_{2}\left(u_{n}\right) & \cdots & \underline{v}_{n}\left(u_{n}\right)
\end{array}\right)
$$


We check the top row: Of course we have $\underline{v}_{1}(\pi)=v_{1}(\pi)=1$. This holds for any uniformizer, so we may use $\pi:=\pi_{1}$. Thus, in terms of Equation 3.3 for $f:=\pi$ we have $f_{1}=\pi$ and then $f_{2}:=\overline{\pi \pi^{-v_{1}(\pi)}}=1$. But if $f_{p}=1$ for any $p \geq 2$, we inductively find $f_{p+1}:=\overline{1 \cdot \pi_{p}^{-v_{p}(1)}}=1$. Checking the left column in Equation 3.7 just amounts to observing that $\underline{v}_{1}\left(u_{i}\right)=v_{1}\left(u_{i}\right)=1$ since $u_{i} \in \mathcal{O}_{v_{1}}^{\times}$. Thus,

$$
\operatorname{det}\left(\begin{array}{cccc}
\underline{v}_{1}(\pi) & \underline{v}_{2}(\pi) & \cdots & \underline{v}_{n}(\pi) \\
\underline{v}_{1}\left(u_{2}\right) & \underline{v}_{2}\left(u_{2}\right) & & \underline{v}_{n}\left(u_{2}\right) \\
\vdots & & \ddots & \vdots \\
\underline{v}_{1}\left(u_{n}\right) & \cdots & & \underline{v}_{n}\left(u_{n}\right)
\end{array}\right)=\operatorname{det}\left(\begin{array}{ccc}
\underline{v}_{2}\left(u_{2}\right) & & \underline{v}_{n}\left(u_{2}\right) \\
& \ddots & \vdots \\
\underline{v}_{2}\left(u_{n}\right) & \cdots & \underline{v}_{n}\left(u_{n}\right)
\end{array}\right) .
$$

We may now use that the residue field $k_{1}$ (as in Diagram 3.1) is an $(n-1)$-DVF. Since $u_{2}, \ldots, u_{n} \in \mathcal{O}_{v_{1}}^{\times}$the rank $(n-1)$ valuation $\underline{v}_{\left[k_{1}\right]}$ of $\overline{u_{i}}$ with respect to $k_{1}$ agrees with the last $n-1$ entries of the rank $n$ valuation $\underline{v}$ which appears on the right in Equation 3.8. To see this, note that if we start the inductive definition of the valuation vector in Equation 3.3, its second term is

$$
f_{1}:=u_{i} \quad f_{2}:=\overline{u_{i} \pi_{p}^{-v_{1}\left(u_{i}\right)}}=\overline{u_{i}}
$$

and then the next inductive steps towards $f_{3}, f_{4}, \ldots$ agree with whether we perform them with respect to $F$ or with respect to $k_{1}$, just differing by an indexing shift. By our inductive hypothesis, the proposition is already proven for all $(n-1)$-DVFs, so we obtain.

$$
\left(\partial_{v_{n}} \cdots \partial_{v_{2}}\right)\left\{\overline{u_{2}}, \ldots, \overline{u_{n}}\right\}=\operatorname{det}\left(\begin{array}{ccc}
\underline{v}_{2}\left(u_{2}\right) & & \underline{v}_{n}\left(u_{2}\right) \\
& \ddots & \vdots \\
\underline{v}_{2}\left(u_{n}\right) & \cdots & \underline{v}_{n}\left(u_{n}\right)
\end{array}\right) .
$$

Thus, $\left(\partial_{v_{n}} \cdots \partial_{v_{1}}\right)\left\{\pi, u_{2}, \ldots, u_{n}\right\}=\left(\partial_{v_{n}} \cdots \partial_{v_{2}}\right)\left\{\overline{u_{2}}, \ldots, \overline{u_{n}}\right\}$ agrees with the right hand side. This finishes the proof.

Corollary 3.10. The determinant on the right-hand side is independent of the choice of uniformizers $\pi_{i} \in \mathcal{O}_{v_{i}}$.

Corollary 3.10 follows immediately from Proposition 3.9 since the left-hand side is independent of the choice of uniformizers.

Definition 3.11. Let $X / k$ be an irreducible smooth proper variety of dimension $n$. Let $Y$ • be a flag in $X$ in the sense of Definition 1.1, not necessarily admissible. Let

$$
\mathcal{G}\left(Y_{\bullet}\right)
$$

be the set of all $n-D V F$ structures on the function field $k(X)$

$$
\begin{aligned}
& k(X) \\
& \mathcal{O}_{w_{1}} \rightarrow k_{\uparrow} \\
& \mathcal{O}_{w_{2}} \rightarrow k_{2}, \\
& \uparrow
\end{aligned}
$$

with the following properties: 
(1) The first valuation ring $\mathcal{O}_{w_{1}} \subseteq k(X)$ satisfies

$$
\mathcal{O}_{X, Y_{1}} \subseteq \mathcal{O}_{w_{1}} \subseteq k(X)
$$

and is of the following form: The ring $\mathcal{O}_{w_{1}}$ is the localization of the integral closure of the left ring inside $k(X)$ at any of its maximal ideals.

(2) For $i \geq 2$, the $i$-th valuation ring in Figure 3.9 satisfies

$$
\mathcal{O}_{Y_{i-1}, Y_{i}} \subseteq \mathcal{O}_{w_{i}} \subseteq \kappa\left(w_{i-1}\right)
$$

and is of the following form: The ring $\mathcal{O}_{w_{i}}$ is the localization of the integral closure of the left ring inside $\kappa\left(w_{i-1}\right)$ at any of its maximal ideals.

Remark 3.12. If $Y_{\bullet}$ happens to be an admissible flag, all the local rings $\mathcal{O}_{Y_{i-1}, Y_{i}}$ are localizations of $\mathcal{O}_{Y_{i-1}, Y_{0}}$ and therefore regular. Hence, in this case the set $\mathcal{G}\left(Y_{\bullet}\right)$ contains only a single element.

Remark 3.13. By the finiteness of integral closure under these assumptions (essentially finite type over a field), it follows that $\mathcal{G}\left(Y_{\bullet}\right)$ is always a finite set.

Example 3.14. Let $C$ be the nodal cubic with equation $y^{2}-x^{2}(x+1)=0$ in $\mathbb{A}_{\mathbb{C}}^{2}$. Then the flag $Y_{\bullet}: \mathbb{A}_{\mathbb{C}}^{2} \supset C \supset(0,0)$ in $\mathbb{A}_{\mathbb{C}}^{2}$ is not admissible. The set $\mathcal{G}\left(Y_{\bullet}\right)$ will consist of two elements. In the usual embedded resolution $R$ of $C \hookrightarrow \mathbb{A}_{\mathbb{C}}^{2}$, let $\tilde{C}$ be the resolved curve (to get this resolution, just blow up the origin). Here $\tilde{C} \rightarrow C$ is the normalization. In the resolution the point $(0,0)$ will have two preimages $p_{1}, p_{2}$. The admissible flags $R \supset \tilde{C} \supset p_{i}$ with $i=1,2$ in the resolution $R$ give rise to the 2-DVF structures on $k(X)$ in $\mathcal{G}\left(Y_{\bullet}\right)$. This is probably the simplest example with cardinality $\# \mathcal{G}\left(Y_{\bullet}\right)>1$.

\subsection{Global computations.}

Theorem 3.15. Let $X / k$ be an irreducible smooth projective variety of dimension $n$. Let $\mathfrak{U}=\left(U_{\alpha}\right)_{\alpha \in I}$ be a finite open cover in the Zariski topology. Suppose

$$
X=\bigcup_{\alpha \in I} \Sigma_{\alpha} \quad \text { with } \quad \Sigma_{\alpha} \subseteq U_{\alpha}
$$

is a disjoint decomposition as a set. Let $D$ be an ample divisor and suppose that $\mathcal{O}_{X}(D)$ is locally given by the Čech cocycle $\left(h_{\alpha}\right)_{\alpha \in I}$ in $\check{H}^{1}\left(\mathfrak{U}, \mathcal{K}_{X}^{\times} / \mathcal{O}_{X}^{\times}\right)$. Let $\underline{v}: k(X)^{\times} \rightarrow \mathbb{Z}_{\text {lex }}^{n}$ be a top rank valuation. Then the volume of the Newton-Okounkov body is given by the formula

$$
\begin{aligned}
\operatorname{Vol} \Delta_{\underline{v}}(D)=\sum_{Y_{\bullet}} \sum_{\left(w_{n}, \ldots, w_{1}\right) \in \mathcal{G}\left(Y_{\bullet}\right)} \sum_{c=0}^{n}(-1)^{c}\left[k\left(w_{n}\right): k\right] & \\
& \frac{1}{n !} \operatorname{det}\left(\begin{array}{ccccc}
\underline{w}_{1}\left(h_{\alpha\left(Y_{0}\right)}\right) & \cdots & \underline{w}_{1} \widehat{\left(h_{\alpha\left(Y_{c}\right)}\right)} & \cdots & \underline{w}_{1}\left(h_{\alpha\left(Y_{n}\right)}\right) \\
\underline{w}_{2}\left(h_{\alpha\left(Y_{0}\right)}\right) & \ddots & & & \underline{w}_{2}\left(h_{\alpha\left(Y_{n}\right)}\right) \\
\vdots & & & & \vdots \\
\underline{w}_{n}\left(h_{\alpha\left(Y_{0}\right)}\right) & \cdots & \underline{w}_{n} \widehat{\left(h_{\alpha\left(Y_{c}\right)}\right)} & \cdots & \underline{w}_{n}\left(h_{\alpha\left(Y_{n}\right)}\right)
\end{array}\right),
\end{aligned}
$$

where

(1) $Y$ • runs through all flags in $X$, but only finitely many will contribute a non-zero summand,

(2) the finite set $\mathcal{G}\left(Y_{\bullet}\right)$ is the one of Definition 3.11 .

(3) $\alpha(Y) \in I$ denotes the unique index such that the generic point of $Y$ is contained in $\Sigma_{\alpha}$ of Equation 3.10 . 
It may or may not happen that the flag $Y_{\bullet}^{\prime}$ appears among those $Y_{\bullet}$ with a non-zero contribution to the sum. Since we can change $Y_{\bullet}^{\prime}$ without changing the Čech cocycle, one can easily produce an example for either situation by adapting $Y_{\bullet}^{\prime}$ as needed.

Proof. (Step 1) We begin with Theorem A of [LM09, i.e.

$$
\operatorname{Vol} \Delta_{\underline{v}}\left(\mathcal{O}_{X}(D)\right)=\lim _{m \rightarrow \infty} \frac{\operatorname{dim}_{k} H^{0}\left(X, \mathcal{O}_{X}(m D)\right)}{m^{n}} .
$$

In fact, the cited theorem only shows this in the special case where $\underline{v}$ comes from an admissible flag in the sense of Definition 1.1. However, it is true for general $\underline{v}$. This is explained in $\mathrm{CFK}^{+}$17, Remark 2.12]: It suffices to work with a birational modification

$$
f: \tilde{X} \longrightarrow X
$$

where $\underline{v}$ comes from an admissible flag. Such an $\tilde{X}$ exists by a type of embedded resolution along the centers of the valuation, see $\left[\mathrm{CFK}^{+} 17\right.$, Theorem 2.9] for the precise result. Finally, the term on the right in Equation 3.11 is a birational invariant, i.e. $H^{0}\left(\tilde{X}, f^{*} \mathcal{O}_{X}(m D)\right)$ has the same growth, Laz04, Proposition 2.2.43], and here Theorem A of LM09 applies verbatim. Note that the definition of $\Delta_{\underline{v}}$ only depends on the valuation on the function field, so it does not see the change of the birational model. As $D$ is ample, the asymptotic Riemann-Roch theorem implies that

$$
\chi\left(\mathcal{O}_{X}(m D)\right)=\frac{m^{n}}{n !} \int_{X} c_{1}\left(\mathcal{O}_{X}(D)\right)^{n}+\mathrm{O}\left(m^{n-1}\right),
$$

see [Laz04, Theorem 1.1.24]. By Serre vanishing for ample line bundles for $m \gg 0$ sufficiently big, the higher cohomology groups in the Euler characteristic vanish, so in the limit in Equation 3.11 simplifies to

$$
\operatorname{Vol} \Delta_{\underline{v}}\left(\mathcal{O}_{X}(D)\right)=\lim _{m \rightarrow \infty} \frac{\chi\left(\mathcal{O}_{X}(m D)\right)}{m^{n}}=\frac{1}{n !} \int_{X} c_{1}\left(\mathcal{O}_{X}(D)\right)^{n}
$$

By the way, instead of the above argument for birational invariance of the volume, we could alternatively compute the intersection number on the right in the modification $\tilde{X}$; this circumvents the admissible flag problem in a different way. The reader may now forget about $\underline{v}$ and $\tilde{X}$; they will not play a rôle in the rest of the proof.

(Step 2) Next, under the connecting map $\delta$ in

$$
H^{0}\left(X, \mathcal{K}_{X}^{\times}\right) \longrightarrow \underset{\left(h_{\alpha}\right)_{\alpha}}{H^{0}}\left(X, \mathcal{K}_{X}^{\times} / \mathcal{O}_{X}^{\times}\right) \stackrel{\delta}{\longrightarrow} \underset{\left(f_{\alpha, \beta}\right)_{\alpha, \beta}}{H^{1}\left(X, \mathcal{O}_{X}\right)} \longrightarrow 0
$$

we get a Cech 1-cocycle representative for the isomorphism class of the invertible sheaf $\mathcal{O}_{X}(D)$ attached to the Cartier divisor; we write $\left(f_{\alpha, \beta}\right)_{\alpha, \beta \in I}$. We use Theorem 2.11 to compute the top self-intersection number of $D$. We can use the same open cover $\mathfrak{U}$ and simply let $f_{\alpha, \beta}^{p}:=f_{\alpha, \beta}$ for $p=1, \ldots, n$, indifferently the value of $p$. We get $\frac{1}{n !} \int_{X} c_{1}\left(\mathcal{O}_{X}(D)\right)^{n}=$

$$
=\frac{1}{n !} \sum_{Y_{\bullet}}\left[k\left(Y_{n}\right): k\right]\left(\partial_{Y_{n}}^{Y_{n-1}} \cdots \partial_{Y_{1}}^{Y_{0}}\right)\left\{f_{\alpha\left(Y_{0}\right) \alpha\left(Y_{1}\right)}, f_{\alpha\left(Y_{1}\right) \alpha\left(Y_{2}\right)}, \ldots, f_{\alpha\left(Y_{n-1}\right) \alpha\left(Y_{n}\right)}\right\}
$$

where the meaning of $Y_{\bullet}, \alpha(-)$ is as in the cited theorem. Each $\partial_{Y_{p+1}}^{Y_{p}}$ by its construction is a sum

$$
\partial_{Y_{p+1}}^{Y_{p}}=\sum_{i} N_{\kappa\left(v_{i}\right) / k\left(Y_{p+1}\right)} \circ \partial_{v_{i}}
$$


for a finite number of discrete valuations $v_{i}$ on $k\left(Y_{p}\right)$; we refer to $\$ 2.3$ where we had recalled this in detail. Since the relevant valuations and the indexing set for $i$ both depend on $Y_{p}$, let us store this extra data in the notation and rewrite the above expression as

$$
\partial_{Y_{p+1}}^{Y_{p}}=\sum_{i_{p} \in J^{(p)}} N_{\kappa\left(v_{i_{p}}^{(p)}\right) / k\left(Y_{p+1}\right)} \circ \partial_{v_{i_{p}}^{(p)}}
$$

where $J^{(p)}$ is a finite index set. We apologize for the heavy notation, we shall not employ it for long. The term $\partial_{Y_{n}}^{Y_{n-1}} \cdots \partial_{Y_{1}}^{Y_{0}}$ in Equation 3.13 unravels as

$$
\begin{aligned}
& \partial_{Y_{n}}^{Y_{n-1}} \cdots \partial_{Y_{1}}^{Y_{0}}=\sum_{i_{n-1} \in J^{(n-1)}} \cdots \sum_{\left.i_{1} \in J^{(1)}\right)} \sum_{i_{0} \in J^{(0)}} \\
& N_{\kappa\left(v_{i_{n-1}}^{(n-1)}\right) / k\left(Y_{n}\right)} \partial_{v_{i_{n-1}}^{(n-1)}} \circ \cdots \circ N_{\kappa\left(v_{i_{1}}^{(1)}\right) / k\left(Y_{2}\right)} \partial_{v_{i_{1}}^{(1)}} \circ N_{\kappa\left(v_{i_{0}}^{(0)}\right) / k\left(Y_{1}\right)} \partial_{v_{i_{0}}^{(0)}}
\end{aligned}
$$

or perhaps more briefly

$$
=\sum_{i_{n-1} \in J^{(n-1)}} \cdots \sum_{i_{1} \in J^{(1)}} \sum_{i_{0} \in J^{(0)}} \prod_{p=0}^{n-1} N_{\kappa\left(v_{i_{p}}^{(p)}\right) / k\left(Y_{p+1}\right)} \circ \partial_{v_{i_{p}}^{(p)}}
$$

if we agree to read the product as the (non-commutative) concatenation of morphisms and unravel it from right to left as $p$ increases. In fact, this is a little better than being plainly non-commutative since the maps originate and end in different objects, so in a certain sense no actual ambiguity is possible.

(Step 3) The expression in Equation 3.14 may, structurally, be summarized as

$$
\underbrace{N \partial N \partial \cdots N \partial}_{n \text { pairs }}=N \underbrace{(\partial N) \cdots(\partial N)(\partial N)}_{(n-1) \text { pairs }} \partial \text {, }
$$

i.e. an alternating composition of boundary maps and norm maps. Now, perform inductively starting from the right the following operation: For each pair $\partial N$ we use Proposition 2.5 and write it as

$$
\partial_{v_{i_{p-1}}^{(p-1)}} N_{\kappa\left(v_{i_{p}}^{(p)}\right) / k\left(Y_{p+1}\right)}=\sum_{w} N_{\kappa(w) / \kappa\left(v_{i_{p-1}}\right)} \partial_{w},
$$

where the sum on the right runs through the finitely many extensions of the valuation $v_{i_{p-1}}^{(p-1)}$ to the finite field extension $\kappa\left(v_{i_{p}}^{(p)}\right)$. We neglect that $w$ itself of course again depends on what $p$ we use on the left side. We arrive at an expression of the shape

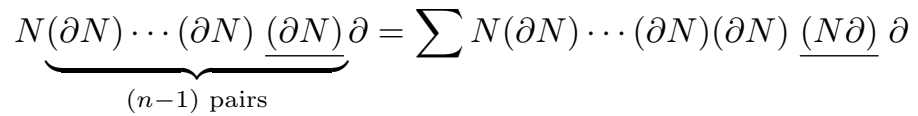

and let us not make precise what the sum on the right side is summing over (it would be the $w)$, and we have underlined the part of the expression where something has changed. The two consecutive norm maps can be combined to one, see Equation 2.5. We get

$$
=\sum N \underbrace{(\partial N) \cdots(\partial N)(\partial N)}_{(n-2) \text { pairs }} \frac{(\partial)}{\partial},
$$

where the underlined expression is what remains from our modifications. The underbraced term has exactly the same shape as what we had started from in Equation 3.15. Inductively 
repeat the procedure, working from right to left. We end up with an expression of the type

$$
=\left(\sum \cdots \sum\right) N \underbrace{\partial \partial \cdots \partial}_{n}
$$

with a single norm map and from each reduction step some finite sum remains. It corresponds in each application of Proposition 2.5 to a choice of the finitely many extensions of the valuation in question. It is messy to spell this out since in each of our reduction steps the valuations change, as we repeatedly switch to a finite field extension. Let us simply write

$$
=\sum_{\mathcal{F}\left(Y_{\bullet}\right)} N \underbrace{\partial \partial \cdots \partial}_{n}
$$

where

$$
\mathcal{F}\left(Y_{\bullet}\right)=\left\{\left(w_{n}, \ldots, w_{1}\right)\right\}
$$

is the finite set of all choices of valuations arising from running the above procedure for the flag $Y_{\bullet}$. It is a little messy to unravel what the elements of $\mathcal{F}\left(Y_{\bullet}\right)$ are in a more explicit fashion (e.g., each $w_{i}$ is a rank one discrete valuation, but describing the field it is defined on is already a little tricky). We will postpone describing $\mathcal{F}\left(Y_{\bullet}\right)$ in more detail.

(Step 4) Return to Equation 3.13 . We obtain $\frac{1}{n !} \int_{X} c_{1}\left(\mathcal{O}_{X}(D)\right)^{n}=$

$$
\begin{aligned}
& \frac{1}{n !} \sum_{Y_{\bullet}}\left[k\left(Y_{n}\right): k\right] \\
& \quad \sum_{\left(w_{n}, \ldots, w_{1}\right) \in \mathcal{F}\left(Y_{\bullet}\right)} N_{\kappa\left(w_{n}\right) / k\left(Y_{n}\right)} \partial_{w_{n}} \cdots \partial_{w_{2}} \partial_{w_{1}}\left\{f_{\alpha\left(Y_{0}\right) \alpha\left(Y_{1}\right)}, f_{\alpha\left(Y_{1}\right) \alpha\left(Y_{2}\right)}, \ldots, f_{\alpha\left(Y_{n-1}\right) \alpha\left(Y_{n}\right)}\right\}
\end{aligned}
$$

and using Equation 2.5 as well as using that on $K_{0}^{\mathrm{M}}$ the norm is multiplication with the field extension degree (see Equation 2.4) once more, this simplifies to

$$
\frac{1}{n !} \sum_{Y_{\bullet}\left(w_{n}, \ldots, w_{1}\right) \in \mathcal{F}\left(Y_{\bullet}\right)}\left[k\left(w_{n}\right): k\right] \partial_{w_{n}} \cdots \partial_{w_{1}}\left\{f_{\alpha\left(Y_{0}\right) \alpha\left(Y_{1}\right)}, f_{\alpha\left(Y_{1}\right) \alpha\left(Y_{2}\right)}, \ldots, f_{\alpha\left(Y_{n-1}\right) \alpha\left(Y_{n}\right)}\right\} .
$$

We can rewrite this in terms of the Cartier divisor Cech representatives $\left(h_{\alpha}\right)_{\alpha}$ of Equation 3.12, giving

$$
\left\{f_{\alpha\left(Y_{0}\right) \alpha\left(Y_{1}\right)}, \ldots, f_{\alpha\left(Y_{n-1}\right) \alpha\left(Y_{n}\right)}\right\}=\sum_{c=0}^{n}(-1)^{c}\left\{h_{\alpha\left(Y_{0}\right)}, \ldots, \widehat{h_{\alpha\left(Y_{c}\right)}}, \ldots, h_{\alpha\left(Y_{n}\right)}\right\}
$$

(we refer to Elaboration 3.16 after the proof for details if this was too quick). Thus,

$$
=\frac{1}{n !} \sum_{Y_{\bullet}} \sum_{\left(w_{n}, \ldots, w_{1}\right) \in \mathcal{F}\left(Y_{\bullet}\right)} \sum_{c=0}^{n}(-1)^{c}\left[k\left(w_{n}\right): k\right] \partial_{w_{n}} \cdots \partial_{w_{1}}\left\{h_{\alpha\left(Y_{0}\right)}, \ldots, \widehat{h_{\alpha\left(Y_{c}\right)}}, \ldots, h_{\alpha\left(Y_{n}\right)}\right\}
$$


Finally, by Proposition 3.9 and switching to the transpose matrix (which does not affect the determinant), we obtain

$$
\begin{aligned}
& =\sum_{Y_{\bullet}} \sum_{\left(w_{n}, \ldots, w_{1}\right) \in \mathcal{F}\left(Y_{\bullet}\right)} \sum_{c=0}^{n}(-1)^{c}\left[k\left(w_{n}\right): k\right] \\
& \frac{1}{n !} \operatorname{det}\left(\begin{array}{ccccc}
\underline{w}_{1}\left(h_{\alpha\left(Y_{0}\right)}\right) & \cdots & \underline{w}_{1} \widehat{\left(h_{\alpha\left(Y_{c}\right)}\right)} & \cdots & \underline{w}_{1}\left(h_{\alpha\left(Y_{n}\right)}\right) \\
\underline{w}_{2}\left(h_{\alpha\left(Y_{0}\right)}\right) & \ddots & & & \underline{w}_{2}\left(h_{\alpha\left(Y_{n}\right)}\right) \\
\vdots & & & \vdots \\
\underline{w}_{n}\left(h_{\alpha\left(Y_{0}\right)}\right) & \cdots & \underline{w}_{n} \widehat{\left(h_{\alpha\left(Y_{c}\right)}\right)} & \cdots & \underline{w}_{n}\left(h_{\alpha\left(Y_{n}\right)}\right)
\end{array}\right),
\end{aligned}
$$

where $\underline{w}$ is the rank $n$ valuation coming from the $n$-DVF structure induced from the valuations $w_{n}, w_{n-1}, \ldots, w_{1}$.

(Step 5) It remains to identify the mysterious set $\mathcal{F}\left(Y_{\bullet}\right)$ from Equation 3.17. We claim that $\mathcal{F}\left(Y_{\bullet}\right)=\mathcal{G}\left(Y_{\bullet}\right)$. Definition 3.11 of $\mathcal{G}\left(Y_{\bullet}\right)$ is inductive in nature: It begins with choosing $w_{1}$ and then going down to pick $w_{i}$ on the basis of $w_{i-1}$. However, the choice of the valuations in $\mathcal{F}\left(Y_{\bullet}\right)$ in the above proof is also inductive in the same way. Hence, it suffices to show that the inductive steps match (it is easy to see that the choice of $w_{1}$ is done in the same way). To this end, we return to Equation 3.16, where we had defined that the next valuation $w_{i}$ "runs through the finitely many extensions of the valuation $v_{i_{p-1}}^{(p-1)}$ to the finite field extension $\kappa\left(v_{i_{p}}^{(p)}\right)$ ". In this step of the proof this is followed by one more map $\partial$ and one more map $N$. Check that in the definition of $\partial$ we pick a valuation from the maximal ideals of the integral closure ${ }^{2}$ (see $\$ 2.3$ where this is carefully explained), and in the norm map, we also pick a valuation from a maximal ideal of the integral closure ${ }^{3}$ (see Proposition 2.5. where this originates from). However, instead of taking these two consecutive integral closures, we may right away only do the second, giving the same outcome. We arrive at the same conditions as in Definition 3.11.

Elaboration 3.16. We provide additional details for the computation in Equation 3.19. The exact sequence of Zariski sheaves $0 \rightarrow \mathcal{O}_{X}^{\times} \rightarrow \mathcal{K}_{X}^{\times} \rightarrow \mathcal{K}_{X}^{\times} / \mathcal{O}_{X}^{\times} \rightarrow 0$ induces the connecting homomorphism $\delta$ in

$$
H^{0}\left(X, \mathcal{K}_{X}^{\times} / \mathcal{O}_{X}^{\times}\right) \stackrel{\delta}{\longrightarrow} H^{1}\left(X, \mathcal{O}_{X}^{\times}\right)
$$

It sends $\left(h_{\alpha}\right)_{\alpha}$ to $\left(f_{\alpha \beta}\right)_{\alpha, \beta}$ with $f_{\alpha \beta}:=h_{\beta} / h_{\alpha}^{-1}$, and if we use Čech representatives on some open cover $\mathfrak{U}$ for $h$, we can still use the same cover for $f$. We compute

$$
\begin{aligned}
\left\{f_{\alpha\left(Y_{0}\right) \alpha\left(Y_{1}\right)}, \ldots, f_{\alpha\left(Y_{n-1}\right) \alpha\left(Y_{n}\right)}\right\} & =\left\{\frac{h_{\alpha\left(Y_{1}\right)}}{h_{\alpha\left(Y_{0}\right)}}, \ldots, \frac{h_{\alpha\left(Y_{n}\right)}}{h_{\alpha\left(Y_{n-1}\right)}}\right\} \\
& =-\sum_{s_{1}=0,1}(-1)^{d_{1}} h_{\alpha\left(Y_{s_{1}}\right)}\left\{\frac{h_{\alpha\left(Y_{2}\right)}}{h_{\alpha\left(Y_{1}\right)}}, \ldots, \frac{h_{\alpha\left(Y_{n}\right)}}{h_{\alpha\left(Y_{n-1}\right)}}\right\}
\end{aligned}
$$

and inductively repeating the idea of the last term manipulation,

$$
=(-1)^{n} \sum_{s_{1}, \ldots, s_{n} \in\{0,1\}}(-1)^{d_{1}+\cdots+d_{n}}\left\{h_{\alpha\left(Y_{s_{1}}\right)}, h_{\alpha\left(Y_{s_{2}+1}\right)}, \ldots, h_{\alpha\left(Y_{s_{n}+n-1}\right)}\right\} .
$$

As soon as two indices agree, the term is 2-torsion by Lemma 2.3 , so cannot map to a nontrivial element in the integers. Thus, only the selections of $n$ distinct consecutive elements

\footnotetext{
2 integral closure inside the field of fractions of the domain

${ }^{3}$ here it is the integral closure in a finite extension of the field of fractions
} 
$s_{1}, s_{2}+1, \ldots, s_{n}+n-1$ of $0<1<\cdots<n$ among these indices is possibly non-zero. So it suffices if we only consider sequences of the shape $(0, \ldots, 0,1,1, \ldots, 1)$ among the $s_{1}, \ldots, s_{n}$. For $(1,1, \ldots, 1)$ we get the total $\operatorname{sign}(-1)^{n} \cdot(-1)^{n}=+1$ and each time we increase the number of leading zeros, the sign changes. Hence, we obtain

$$
=\sum_{c=0}^{n}(-1)^{c}\left\{h_{\alpha\left(Y_{0}\right)}, \ldots, \widehat{h_{\alpha\left(Y_{c}\right)}}, \ldots, h_{\alpha\left(Y_{n}\right)}\right\}
$$

as required.

Question 1. In our example in $\$ 4$ only two flags contribute. Under what circumstances does only one single flag $Y_{\bullet}$ contribute a non-zero summand in the right side of the equation of Theorem 3.15? Can one give a general criterion? If this flag is admissible, $\mathcal{G}\left(Y_{\bullet}\right)$ contains only one element (Remark 3.12 and one can choose $\underline{v}$ to be this top rank valuation, too. Then the theorem gives an equation only involving one single valuation on both sides of the equation. Can one understand the equality in this case in terms of convex geometry?

\subsection{Proof of the main result.}

Theorem 3.17. Let $X / k$ be an irreducible smooth projective variety of dimension $n$.

(1) Let $\underline{v}: k(X)^{\times} \rightarrow \mathbb{Z}_{\text {lex }}^{n}$ be a top rank valuation.

(2) Let $D$ be an ample divisor.

(3) Suppose the graded semigroup $\Gamma_{\underline{v}}(D)$ is finitely generated.

(4) Choose some $m \geq 1$ sufficiently big so that $m D$ is very ample. Let $\left(U_{\alpha}, f_{\alpha}\right)_{\alpha \in I}$ be the local trivialization of $m D$ as a Cartier divisor on a finite open cover $\left(U_{\alpha}\right)_{\alpha \in I}$ produced by Lemma 1.8 .

(5) Suppose

$$
X=\bigcup_{\alpha \in I} \Sigma_{\alpha} \quad \text { with } \quad \Sigma_{\alpha} \subseteq U_{\alpha}
$$

is a disjoint decomposition as a set.

Then

$$
\begin{aligned}
& \operatorname{Vol}\left(\underset{\alpha \in I}{\operatorname{convex}} \operatorname{hull}\left(\frac{1}{m} \underline{v}\left(h_{\alpha}\right)\right)\right)= \\
& \qquad \sum_{Y_{\bullet}} \sum_{\left(w_{n}, \ldots, w_{1}\right) \in \mathcal{G}\left(Y_{\bullet}\right)} \sum_{c=0}^{n}(-1)^{c}\left[k\left(w_{n}\right): k\right] \\
& \quad \operatorname{Vol} \operatorname{simplex}\left\langle\underline{w}\left(h_{\alpha\left(Y_{0}\right)}\right), \ldots \underline{w} \widehat{\left(h_{\alpha\left(Y_{c}\right)}\right)} \ldots, \underline{w}\left(h_{\alpha\left(Y_{n}\right)}\right)\right\rangle
\end{aligned}
$$

and both values are the volume of the Newton-Okounkov body $\mathrm{Vol}_{Y_{\mathbf{0}}}(D)$. The first sum runs over all flags $Y_{\bullet}$ in the sense of Definition 1.1 and for each flag $\mathcal{G}\left(Y_{\bullet}\right)$ is as in Definition 3.11,

Remark 3.18. The right-hand side is independent of the choice of $\underline{v}$.

We point out that by "volume of the simplex" we mean the signed volume, i.e. if the vectors are in the opposite orientation to the standard basis of $\mathbb{R}^{n}$, the volume is accordingly a negative value - see Equations 0.1 and 0.2 . 
Proof. Use Theorem 3.15. Then express $\operatorname{Vol} \Delta_{\underline{v}}(D)$ using the presentation as a convex polytope coming from Lemma 1.8 . We obtain

$$
\operatorname{Vol}\left(\underset{\alpha \in I}{\operatorname{convex} \operatorname{hull}}\left(\frac{1}{m} \underline{v}\left(h_{\alpha}\right)\right)\right)=\operatorname{Vol} \Delta_{\underline{v}}(D)
$$

and this in turn equals

$$
=\sum_{Y_{\bullet}} \sum_{\left(w_{n}, \ldots, w_{1}\right) \in \mathcal{F}\left(Y_{\bullet}\right)} \sum_{c=0}^{n}(-1)^{c}\left[k\left(w_{n}\right): k\right] \frac{1}{n !} \operatorname{det}\left(\left[\underline{w}_{\ell}\left(h_{\alpha\left(Y_{m}\right)}\right)\right] \begin{array}{c}
\ell=1, \ldots, n \\
m=0, \ldots, \widehat{c}, \ldots, n
\end{array}\right),
$$

but for $n$ vectors $\underline{x}_{i} \in \mathbb{R}^{n}$, the expression $\frac{1}{n !} \operatorname{det}\left(\underline{x}_{1}, \ldots, \underline{x}_{n}\right)$ is the (signed) volume of the (oriented) $n$-simplex in $\mathbb{R}^{n}$ spanned by the vectors $\underline{x}_{1}, \ldots, \underline{x}_{n}$. This is clear: Without the factor $n$ ! it is just the volume of the spanned parallelepiped.

\section{A fully WORKed out EXAmple}

In this section we will present a detailed example demonstrating our main formula. Our example will depend on three parameters $l \in \mathbb{Z}_{>1}, a, b \in \mathbb{Z}$, the first indicates running through an infinite family of surfaces, while $a, b$ allow us to run through an infinite family of divisors on them. Hence, in a sense, we discuss a countably infinite set of examples.

Consider the Hirzebruch surface $F_{l}$. This is a toric surface, and this is the viewpoint we shall exploit . We will use the notation of Fulton [Ful93]. Let $N:=\mathbb{Z}^{2}$. The polyhedral fan $\Sigma$ in question is the following one.

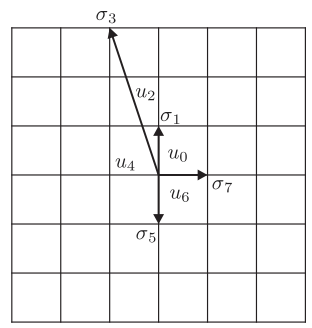

We write $u_{0}, \ldots, u_{6}$ with even indices to denote the 2 -dimensional cones, $\sigma_{1}, \ldots, \sigma_{7}$ with odd indices to denote the 1-dimensional cones and $\eta$ will denote the origin. This unusual indexing is used so that we have

the cone $u_{i}$ has the two facets $\sigma_{i-1}$ and $\sigma_{i+1}$

for all $i$ (tacitly we set $\sigma_{-1}:=\sigma_{7}$ ). The ray $\sigma_{3}$ ends at the point $(-1, l)$. Write $X(\Sigma)$ for the toric variety attached to a polyhedral fan $\Sigma$. Write $\Sigma \subseteq N_{\mathbb{R}}$ for the polyhedral fan of Figure 4.1, and in brief $X:=X(\Sigma)$. Our $X$ is an integral smooth projective toric surface. We have $\operatorname{Pic}(X) \cong \mathbb{Z}\left\langle\sigma_{1}, \sigma_{3}\right\rangle$. Consider the divisor

$$
D=a \sigma_{1}+b \sigma_{3} \quad \text { for } \quad a, b \in \mathbb{Z} .
$$

We claim that the divisor $D$ is ample if and only if

$$
a>0 \quad \text { and } \quad b-l a>0 .
$$

This is not too hard to see, but this computation is also carried out in CLS11, Example 6.1.16], use that in the notation loc. cit. our $D$ would be $a D_{4}+(b-l a) D_{3}$, giving our claim here.

\footnotetext{
${ }^{4}$ One could also view $F_{l}$ as a projective bundle over $\mathbb{P}^{1}$, but this perspective seems less convenient when setting up the open covers of Theorem 3.17
} 
4.1. Step 1: Local equations for the Cartier divisor. We will be aiming towards Theorem 3.17, so we need to fix a trivialization for the line bundle $\mathcal{O}_{X}(D)$ in some open cover. To this end, we shall use the standard affine opens $U_{i}:=X\left(u_{i}\right)$ for $i \in I:=\{0,2,4,6\}$ from the toric theory as our Cech cover $\mathfrak{U}$ of $X$. This motivates why we denote the topdimensional cones by the letter $u$. Let us compute the Cartier divisor representative of $D \in H^{0}\left(X, \mathcal{K}_{X}^{\times} / \mathcal{O}_{X}^{\times}\right)$. We write $D=\left(h_{\alpha}\right)_{\alpha \in I}$, and abstractly $D=\sum d_{i} \sigma_{i}$ for odd $i$ and $d_{i} \in \mathbb{Z}$ (i.e. $d_{5}=d_{7}=0$ ). For each $i \in I$ this means that we need to solve the equations

$$
\left\langle h_{i}, \sigma_{i-1}\right\rangle=-d_{i-1} \quad \text { and } \quad\left\langle h_{i}, \sigma_{i+1}\right\rangle=-d_{i+1}
$$

because $\sigma_{i-1}, \sigma_{i+1}$ are the facets of the cone $u_{i}$ by our notational convention from Equation 4.2. [Ful93, p. 61, Lemma]. Since the cone is smooth, these rays form a vector space basis of $N_{\mathbb{R}}$. For example, for $i=2$ and if we write $x^{\xi} y^{\psi}$ for the monomial exponents, we have to solve the equations

$$
\begin{array}{ll}
\left(\begin{array}{c}
\xi \\
\psi
\end{array}\right) \sigma_{1}=-a \quad \text { that is } & \left(\begin{array}{l}
\xi \\
\psi
\end{array}\right) \cdot\left(\begin{array}{l}
0 \\
1
\end{array}\right)=\psi=-a \\
\left(\begin{array}{c}
\xi \\
\psi
\end{array}\right) \sigma_{3}=-b \quad \text { that is } & \left(\begin{array}{c}
\xi \\
\psi
\end{array}\right) \cdot\left(\begin{array}{c}
-1 \\
l
\end{array}\right)=-\xi+\psi l=-b
\end{array}
$$

and therefore $h_{2}:=x^{b-l a} y^{-a}$. We leave the rest of this computation (i.e. $h_{0}, h_{4}, h_{6}$ ) to the reader. The result is

$$
h_{0}:=y^{-a} \quad h_{2}:=x^{b-l a} y^{-a} \quad h_{4}:=x^{b} \quad h_{6}:=1 .
$$

Thus, the Cech 1-cocycle for the line bundle class of $\mathcal{O}_{X}(D)$ in $\operatorname{Pic}(X) \cong H^{1}\left(X, \mathcal{O}_{X}^{\times}\right)$is given by $\left(f_{\alpha \beta}\right)$ and $f_{\alpha \beta}:=h_{\beta} h_{\alpha}^{-1}$ (this corresponds to what we had discussed in Elaboration 3.16).

Remark 4.1. These computations also give the divisor polytope $P_{D}$ (as explained in Ful93, p. 66]). Its defining inequalities are those of Equation 4.4, just replace "=" with " $\geq$ ". If $D$ is ample (cf. Equation 4.3), we obtain the polytope

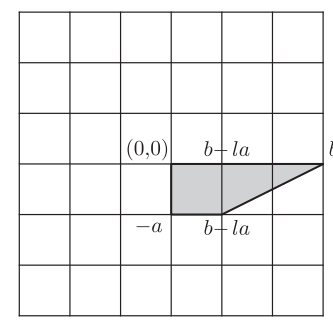

which has Euclidean volume $(b-l a) a$ (for the box on the left) and $\frac{1}{2} l a^{2}$ (for the triangle on the right), and so in total $b a-\frac{1}{2} l a^{2}$. By [LM09, Proposition 6.1, (i)] the Newton-Okounkov body $\Delta_{Y_{\bullet}}(D)$ for a suitable flag (see loc. cit.) agrees with $P_{D}$, so we learn that

$$
\frac{1}{2 !} \operatorname{Vol}_{\text {birat }}(D)=\operatorname{Vol}_{\text {Eucl }}\left(P_{D}\right)=a b-\frac{1}{2} l a^{2}
$$

by [LM09, Theorem A].

Example 4.2. Let us double check the validity of this using intersection theory. We use the quick formalism of [Ful93, $§ 2.5$, p. 43, p.44, last exercise]. We have

$$
\begin{array}{rlrl}
l \cdot \sigma_{1} & =\sigma_{7}+\sigma_{3} & 0 \cdot \sigma_{3} & =\sigma_{1}+\sigma_{5} \\
(-l) \cdot \sigma_{5} & =\sigma_{3}+\sigma_{7} & 0 \cdot \sigma_{7} & =\sigma_{1}+\sigma_{5}
\end{array}
$$


and then $\sigma_{1}^{2}=-l$ and $\sigma_{3}^{2}=-0$ in the Chow ring, if we indentify the names of the rays with their divisors. The divisors $\sigma_{5}, \sigma_{7}$ are linearly dependent in the Picard group. Thus,

$$
D^{2}=\left(a \sigma_{1}+b \sigma_{3}\right)=a^{2} \sigma_{1}^{2}+2 a b \sigma_{1} \sigma_{3}+b^{2} \sigma_{3}^{2}=2 a b-l a^{2},
$$

which confirms Equation 4.7 by using the characterization of the birational volume through the asymptotic Riemann-Roch theorem. Again, this is only valid since we have the assumptions of Equation 4.3 in place.

4.2. Step 2: Using the orbit decomposition. In order to apply Theorem 3.17, we need to cook up a disjoint decomposition $\left(\Sigma_{j}\right)_{j \in I}$ of $X$ as a set, as in Equation 3.20. We do this as follows: By the orbit-cone correspondence the scheme $X$ can be written as the disjoint union of its orbits $O(\tau)$, where $\tau$ runs through all cones of the polyhedral fan, Ful93, p. 54] CLS11, Theorem 3.2.6]. Recall that,

$$
U_{\tau}=\coprod_{\mu \leq \tau} O(\mu)
$$

so it will be easy to control $\Sigma_{j} \subseteq U_{j}$ for $j \in I$ if we only build the $\Sigma_{j}$ from disjoint unions of orbits. Recall that $\eta$ is the trivial cone; just the origin. It corresponds to the big open orbit $\mathbb{G}_{m} \times \mathbb{G}_{m}$ in the surface. Our naming convention from Equation 4.2 suggests the following choice:

$$
\begin{aligned}
& \Sigma_{0}:=O(\eta) \amalg O\left(\sigma_{-1}\right) \amalg O\left(u_{0}\right) \subseteq U_{0} \\
& \Sigma_{2}:=O\left(\sigma_{1}\right) \amalg O\left(u_{2}\right) \subseteq U_{2} \\
& \Sigma_{4}:=O\left(\sigma_{3}\right) \amalg O\left(u_{4}\right) \subseteq U_{4} \\
& \Sigma_{6}:=O\left(\sigma_{5}\right) \amalg O\left(u_{6}\right) \subseteq U_{6} .
\end{aligned}
$$

Thus, except for adding the big open orbit $O(\eta)$ to $\Sigma_{0}$, we always just take a rank one $\mathbb{G}_{m^{-}}$ torus $O\left(\sigma_{i-1}\right)$ and a single closed point $O\left(u_{i}\right)$. All orbits are present, so this is a valid disjoint decomposition.

Remark 4.3. The sets $\Sigma_{2}, \Sigma_{4}$ and $\Sigma_{4}$ are the underlying sets of affine lines $\mathbb{A}_{k}^{1}$ in $X$. We will not use this.

\subsection{Computing the right side.}

4.3.1. Reducing to a finite set of flags. Let us compute the right side in Theorem 3.17, that is

$$
\begin{aligned}
\sum_{Y_{\bullet}} \sum_{\left(w_{2}, w_{1}\right) \in \mathcal{G}\left(Y_{\bullet}\right)} \sum_{c=0}^{2}(-1)^{c}\left[k\left(w_{2}\right): k\right] \\
\quad \text { Vol simplex }\left\langle\underline{w}\left(h_{\alpha\left(Y_{0}\right)}\right), \ldots \underline{w} \widehat{\left(h_{\alpha\left(Y_{c}\right)}\right)} \ldots, \underline{w}\left(h_{\alpha\left(Y_{2}\right)}\right)\right\rangle .
\end{aligned}
$$

A priori we have no control which of these uncountably many summands will be non-zero, there is a huge supply of flags $Y_{\bullet}$. This is best approached as follows: For the evaluation of each summand we only need to know the values of $\alpha\left(Y_{i}\right) \in I$ for $i=0,1,2$ and since $I:=\{0,2,4,6\}$ we can make a case distinction depending on what values the $\alpha\left(Y_{i}\right)$ attain. These a priori $4^{3}=64$ cases can quickly be cut down to a manageable number:

Possible values for $\alpha\left(Y_{0}\right)$ : Since $Y_{0}$ is a codimension zero integral closed subscheme of $X$, it can only be all of $X$. The generic point of $X$ lies in $\Sigma_{0}$ by Equation 4.10 . Thus, $\alpha\left(Y_{0}\right)=0$ for all flags. 
Possible values for $\alpha\left(Y_{1}\right)$ : This is more complicated. The variety $Y_{1}$ is a curve in $X$; we have such for all possible values in $I$.

We use a trick: In Equation 4.12 we only get a contribution if the valuation vectors $\underline{w}\left(h_{\alpha\left(Y_{i}\right)}\right)$ are non-zero. Otherwise the spanned 2-simplex is degenerate and has volume zero. But all possible values for $h_{\alpha\left(Y_{i}\right)}$ are those listed in Equation 4.5. These are (locally in the $U_{i}$ ) local equations for the divisors attached to the rays. So, already when computing the first level valuation, i.e. the $v_{1}$ in

$$
\underline{v}(f)=\left(\underline{v}_{1}(f), \underline{v}_{2}(f)\right),
$$

it can only be non-zero at the divisors coming from these rays, i.e. the $T$-invariant divisors on the toric surface. Similarly, all the closed points which form the $Y_{2}$ of a flag, and which determine the second component $\underline{v}_{2}(f)$, can only be possibly non-zero when they lie on such a divisor. Thus, if at least one component of the vector $\underline{v}(f)$ needs to be non-zero, we can restrict for $Y_{1}$ to $T$-invariant divisors; and indeed for $Y_{0}$ to the intersections of such divisors. Let us record this essential simplification as a lemma:

Lemma 4.4. In Equation 4.11 a non-zero summand can only stem from a flag $Y_{\bullet}$ such that

$$
Y_{0}=X \quad Y_{1}=V\left(\sigma_{i}\right) \quad Y_{0}=V\left(u_{j}\right), \quad Y_{0} \supset Y_{1} \supset Y_{2},
$$

for some indices $i, j$ (here for an orbit $\tau$ we write $V(\tau)$ for the orbit closure as in Fulton's book).

Besides cutting down the values of $Y_{\bullet}$ in the first sum in Equation 4.11, we get a few more useful facts from this: All the closed points $V\left(u_{j}\right)$ regardless the index $j$ are $k$-rational, so in Equation 4.11 we have $\left[k\left(w_{n}\right): k\right]=1$ for all flags we need to consider. Furthermore, all these flags are flags of $T$-invariant divisors in a smooth toric variety and therefore admissible, so the set $\mathcal{G}\left(Y_{\bullet}\right)$ contains only a single element, Remark 3.12 (all the local rings in Definition 3.11 are already integrally closed). This also implies that the valuation $\underline{w}$ is really just the one attached to $Y_{\bullet}$ itself.

4.3.2. Eliminating a priori vanishing summands. Our sum has therefore simplified to the finite sum

$$
\left.\sum_{\substack{Y_{\bullet} \\ \text { as in Eq. } 4.13}} \sum_{c=0}^{2}(-1)^{c} \operatorname{Vol} \operatorname{simplex}\left\langle\underline{w}_{Y_{\bullet}}\left(h_{\alpha\left(Y_{0}\right)}\right), \ldots \underline{w}_{Y_{\bullet}} \widehat{\left(h_{\alpha\left(Y_{c}\right)}\right.}\right) \ldots, \underline{w}_{Y_{\bullet}}\left(h_{\alpha\left(Y_{2}\right)}\right)\right\rangle,
$$

where $\underline{w}_{Y_{\bullet}}$ is the valuation attached to $Y_{\bullet}$. The summation over $c$ triples the summands we have to evaluate. We can simplify this: Inspecting Equation 3.18 and Equation 3.19 in the proof underlying our main formula, we see that we get a sum

$$
\frac{1}{n !} \sum_{Y_{\bullet}} \sum_{\left(w_{n}, \ldots, w_{1}\right) \in \mathcal{F}\left(Y_{\bullet}\right)}\left[k\left(w_{n}\right): k\right] \partial_{w_{n}} \cdots \partial_{w_{1}}\left\{f_{\alpha\left(Y_{0}\right) \alpha\left(Y_{1}\right)}, f_{\alpha\left(Y_{1}\right) \alpha\left(Y_{2}\right)}, \ldots, f_{\alpha\left(Y_{n-1}\right) \alpha\left(Y_{n}\right)}\right\}
$$

first - which later gets transformed into a sum of the shape as in Equation 4.14 (see Elaboration 3.16 for details). In our case, the inner term is

$$
\frac{1}{2 !} \sum_{Y_{\bullet}} \partial_{w_{2}} \partial_{w_{1}}\left\{f_{\alpha\left(Y_{0}\right) \alpha\left(Y_{1}\right)}, f_{\alpha\left(Y_{1}\right) \alpha\left(Y_{2}\right)}\right\},
$$

where $\left(f_{\alpha \beta}\right)$ are the local equations for the 1-cocycle of $\mathcal{O}_{X}(D)$, exactly as explained below Equation 4.5. We use the following trick: If for a flag $Y_{\bullet}$ the summand

$$
\partial_{w_{2}} \partial_{w_{1}}\left\{f_{\alpha\left(Y_{0}\right) \alpha\left(Y_{1}\right)}, f_{\alpha\left(Y_{1}\right) \alpha\left(Y_{2}\right)}\right\}
$$


is zero, then upon the rewriting in Elaboration 3.16 the 3 resulting terms necessarily add up to zero, too, so there is no harm in neglecting these flags as summands altogether.

Let us carry out this computation now: We use the indices $i$ and $j$ as introduced in Equation 4.13. Letting columns represent the values of $i$ and rows the values of $j$, we obtain the possible summands; their arguments are

\begin{tabular}{c|cccc} 
& 1 & 3 & 5 & 7 \\
\hline 0 & $\left\{f_{02}, f_{20}\right\}$ & $\left\{f_{04}, f_{40}\right\}$ & $\left\{f_{06}, f_{60}\right\}$ & $\left\{f_{00}, f_{00}\right\}$ \\
2 & $\left\{f_{02}, f_{22}\right\}$ & $\left\{f_{04}, f_{42}\right\}$ & $\left\{f_{06}, f_{62}\right\}$ & $\left\{f_{00}, f_{02}\right\}$ \\
4 & $\left\{f_{02}, f_{24}\right\}$ & $\left\{f_{04}, f_{44}\right\}$ & $\left\{f_{06}, f_{64}\right\}$ & $\left\{f_{00}, f_{04}\right\}$ \\
6 & $\left\{f_{02}, f_{26}\right\}$ & $\left\{f_{04}, f_{46}\right\}$ & $\left\{f_{06}, f_{66}\right\}$ & $\left\{f_{00}, f_{06}\right\}$
\end{tabular}

Most terms must be zero on general grounds: If both indices agree, we have $f_{\alpha \alpha}=1$ because

$$
f_{\alpha \beta}:=h_{\beta} h_{\alpha}^{-1} .
$$

This kills for example the entire right column and a downward diagonal. By the same formula, we also have $\left\{f_{\alpha \beta}, f_{\beta \alpha}\right\}=\left\{f_{\alpha \beta}, f_{\alpha \beta}^{-1}\right\}$ and for any $f,\left\{f, f^{-1}\right\}$ is 2-torsion in $K_{2}^{\mathrm{M}}$ by Lemma 2.3 . so must be mapped to zero under any map to the reals, and therefore cannot contribute non-trivially. This leaves only the following pairs $(i, j)$,

\begin{tabular}{c|cccc} 
& 1 & 3 & 5 & 7 \\
\hline 0 & & & \\
2 & & $\left\{f_{04}, f_{42}\right\}$ & $\left\{f_{06}, f_{62}\right\}$ \\
4 & $\left\{f_{02}, f_{24}\right\}$ & & $\left\{f_{06}, f_{64}\right\}$ \\
6 & $\left\{f_{02}, f_{26}\right\}$ & $\left\{f_{04}, f_{46}\right\}$ &
\end{tabular}

However, many of these index pairs $(i, j)$ cannot occur such that $Y_{\bullet}$ is a flag, i.e. the inclusion condition in Equation 4.13 would be broken. In detail: Note that $\sigma_{i}$ according to Equation 4.10 lies in $\Sigma_{i+1}$, so $\alpha\left(\sigma_{i}\right)=i+1$ (and read $\Sigma_{8}$ as $\Sigma_{0}$ in the case of $\sigma_{7}$ ). Similarly, the single point of the orbit $u_{j}$ lies in $\Sigma_{j}$. Once we are in the orbit $O\left(\sigma_{i}\right)$, then by the orbit-cone correspondence, its closure is

$$
V\left(\sigma_{i}\right)=O\left(\sigma_{i}\right) \amalg O\left(u_{i-1}\right) \amalg O\left(u_{i+1}\right),
$$

by [Ful93, p. 54]. Again, our special indexing comes in handy. We deduce that if $Y_{1}=V\left(\sigma_{i}\right)$, then the closed point $Y_{2}$ of a flag can only lie in one of these three sets.

If it lies on the orbit $O\left(\sigma_{i}\right)$, this means $\alpha\left(Y_{1}\right)=\alpha\left(Y_{2}\right)$ and thus $f_{\alpha\left(Y_{1}\right) \alpha\left(Y_{2}\right)}=1$ by Equation 4.15 implying that the term $\{-,-\}$ is zero. Hence, we may restrict to $Y_{2}$ being the closed points $O\left(u_{i-1}\right)$ or $O\left(u_{i+1}\right)$. Again, by Equation 4.10 if it is $O\left(u_{i+1}\right)$, they still both lie in $\Sigma_{i+1}$, so still $\alpha\left(Y_{1}\right)=\alpha\left(Y_{2}\right)$, and thus $\{-,-\}$ is zero. Thus, only $O\left(u_{i-1}\right)$ is possible if we want a non-zero contribution. Using this constraint, only two possibly non-zero summands

\begin{tabular}{|c|c|c|}
\hline & 3 & 5 \\
\hline 0 & & \\
\hline 2 & $\left\{f_{04}, f_{42}\right\}$ & \\
\hline $\begin{array}{l}4 \\
6\end{array}$ & & $\left\{J_{06}, J_{64}\right\}$ \\
\hline
\end{tabular}
remain:

and these belong to the following flags:

(1) $Y_{\bullet}=\left(X \supset V\left(\sigma_{3}\right) \supset V\left(u_{2}\right)\right)$ with $\alpha\left(Y_{0}\right)=0, \alpha\left(Y_{1}\right)=4, \alpha\left(Y_{2}\right)=2$, and

(2) $Y_{\bullet}=\left(X \supset V\left(\sigma_{5}\right) \supset V\left(u_{4}\right)\right)$ with $\alpha\left(Y_{0}\right)=0, \alpha\left(Y_{1}\right)=6, \alpha\left(Y_{2}\right)=4$. 
The whole point of the paper is the presence of different flags along which we take the span of valuation vectors, and here we have isolated the two critical flags in our example. Next, we return to our original formula in Equation 4.14 - knowing that we only need to evaluate it for these two flags $Y_{\bullet}$.

4.3.3. The simplex attached to $X \supset V\left(\sigma_{3}\right) \supset V\left(u_{2}\right)$. For the flag $X \supset V\left(\sigma_{3}\right) \supset V\left(u_{2}\right)$, we can conveniently compute the valuation vectors in an affine open of $X$. The closed point $V\left(u_{2}\right)$ lies in $U_{2}$ by the orbit-cone correspondence. We easily compute that $U_{2}=\operatorname{Spec} k\left[x^{-1}, x^{l} y\right]$. In this open the local equation for $V\left(\sigma_{3}\right)$ is the principal ideal $\left(x^{-1}\right)$, so this corresponds to the valuation $\underline{w}_{1}$, and $V\left(u_{2}\right)$ is cut out by $\left(x^{-1}, x^{l} y\right)$, so $x^{l} y$ is a uniformizer for the component $\underline{w}_{2}$. Equation 4.14 asks us to compute

$$
\begin{gathered}
\left.\sum_{c=0}^{2}(-1)^{c} \text { Vol simplex }\left\langle\underline{w}_{Y_{\bullet}}\left(h_{\alpha\left(Y_{0}\right)}\right), \ldots \underline{w}_{Y_{\bullet}} \widehat{\left(h_{\alpha\left(Y_{c}\right)}\right.}\right) \ldots, \underline{w}_{Y_{\bullet}}\left(h_{\alpha\left(Y_{2}\right)}\right)\right\rangle \\
=\operatorname{Vol} \operatorname{simplex}\left\langle\underline{w}_{Y_{\bullet}}\left(h_{4}\right), \underline{w}_{Y_{\bullet}}\left(h_{2}\right)\right\rangle-\operatorname{Vol} \operatorname{simplex}\left\langle\underline{w}_{Y_{\bullet}}\left(h_{0}\right), \underline{w}_{Y_{\bullet}}\left(h_{2}\right)\right\rangle \\
\quad+\operatorname{Vol} \operatorname{simplex}\left\langle\underline{w}_{Y_{\bullet}}\left(h_{0}\right), \underline{w}_{Y_{\bullet}}\left(h_{4}\right)\right\rangle .
\end{gathered}
$$

We get

$$
\begin{aligned}
& \text { Vol simplex }\left\langle\underline{w}_{Y_{\bullet}}\left(h_{4}\right), \underline{w}_{Y_{\bullet}}\left(h_{2}\right)\right\rangle=\frac{1}{2} \operatorname{det}\left(\begin{array}{ll}
\underline{w}_{1}\left(x^{b}\right) & \underline{w}_{1}\left(x^{b-l a} y^{-a}\right) \\
\underline{w}_{2}\left(x^{b}\right) & \underline{w}_{2}\left(x^{b-l a} y^{-a}\right)
\end{array}\right) \\
& =\frac{1}{2} \operatorname{det}\left(\begin{array}{ll}
\underline{w}_{1}\left(x^{b}\right) & \underline{w}_{1}\left(\left(x^{l} y\right)^{-a} \cdot x^{b}\right) \\
\underline{w}_{2}\left(x^{b}\right) & \underline{w}_{2}\left(\left(x^{l} y\right)^{-a} \cdot x^{b}\right)
\end{array}\right)=\frac{1}{2} \operatorname{det}\left(\begin{array}{cc}
-b & -b \\
0 & -a
\end{array}\right)=\frac{1}{2} a b .
\end{aligned}
$$

The next 2-simplex is more interesting. Note that $y^{-a}=\left(x^{l} y\right)^{-a} \cdot x^{l a}$ and therefore

$$
\begin{aligned}
& \text { Vol simplex }\left\langle\underline{w}_{Y \bullet}\left(h_{0}\right), \underline{w}_{Y \bullet}\left(h_{2}\right)\right\rangle=\frac{1}{2} \operatorname{det}\left(\begin{array}{ll}
\underline{w}_{1}\left(\left(x^{l} y\right)^{-a} \cdot x^{l a}\right) & \underline{w}_{1}\left(\left(x^{l} y\right)^{-a} \cdot x^{b}\right) \\
\underline{w}_{2}\left(\left(x^{l} y\right)^{-a} \cdot x^{l a}\right) & \underline{w}_{2}\left(\left(x^{l} y\right)^{-a} \cdot x^{b}\right)
\end{array}\right) \\
& =\frac{1}{2} \operatorname{det}\left(\begin{array}{cc}
-l a & -b \\
-a & -a
\end{array}\right)=\frac{1}{2}\left(l a^{2}-a b\right)
\end{aligned}
$$

and finally, Vol simplex $\left\langle\underline{w}_{Y_{\bullet}}\left(h_{0}\right), \underline{w}_{Y_{\bullet}}\left(h_{4}\right)\right\rangle$ equals

$$
=\frac{1}{2} \operatorname{det}\left(\begin{array}{ll}
\underline{w}_{1}\left(\left(x^{l} y\right)^{-a} \cdot x^{l a}\right) & \underline{w}_{1}\left(x^{b}\right) \\
\underline{w}_{2}\left(\left(x^{l} y\right)^{-a} \cdot x^{l a}\right) & \underline{w}_{2}\left(x^{b}\right)
\end{array}\right)=\frac{1}{2} \operatorname{det}\left(\begin{array}{cc}
-l a & -b \\
-a & 0
\end{array}\right)=-\frac{1}{2} a b .
$$

Thus, the total summand for this flag in Equation 4.14 is

$$
\frac{1}{2} a b-\frac{1}{2}\left(l a^{2}-a b\right)+\frac{1}{2}(-a b)=\frac{1}{2} a b-\frac{1}{2} l a^{2} .
$$

4.3.4. The simplex attached to $X \supset V\left(\sigma_{5}\right) \supset V\left(u_{4}\right)$. Now, we need to do the analogous evaluation for this flag, but this turns out to be shorter. We write $\underline{w}^{\prime}$ for the valuation of this flag. The affine open $U_{4}=\operatorname{Spec} k\left[x^{-1}, x^{-l} y^{-1}\right]$ contains $O\left(u_{4}\right)$ and $O\left(\sigma_{5}\right)$ by Equation 4.9. The local equation for the orbit closure $V\left(\sigma_{5}\right)$ is the principal ideal $\left(x^{-1}\right)$. The rank one valuation along this ideal is the first component $\underline{w}_{1}^{\prime}$. Moreover, $x^{-l} y^{-1}$ is a uniformizer for 
the valuation $\underline{w}_{2}^{\prime}$. For this flag, Equation 4.14 adds the contribution from

$$
\begin{gathered}
\sum_{c=0}^{2}(-1)^{c} \text { Vol simplex }\left\langle\underline{w}_{Y_{\bullet}}\left(h_{\alpha\left(Y_{0}\right)}\right), \ldots \underline{w}_{Y_{\bullet}} \widehat{\left.\left(h_{\alpha\left(Y_{c}\right)}\right) \ldots, \underline{w}_{Y_{\bullet}}\left(h_{\alpha\left(Y_{2}\right)}\right)\right\rangle}\right. \\
=\text { Vol simplex }\left\langle\underline{w}_{Y_{\bullet}}\left(h_{6}\right), \underline{w}_{Y_{\bullet}}\left(h_{4}\right)\right\rangle-\operatorname{Vol} \operatorname{simplex}\left\langle\underline{w}_{Y_{\bullet}}\left(h_{0}\right), \underline{w}_{Y_{\bullet}}\left(h_{4}\right)\right\rangle \\
\quad+\operatorname{Vol} \operatorname{simplex}\left\langle\underline{w}_{Y_{\bullet}}\left(h_{0}\right), \underline{w}_{Y_{\bullet}}\left(h_{6}\right)\right\rangle .
\end{gathered}
$$

Note that $h_{6}=1$, so $\underline{w}_{Y_{\bullet}}\left(h_{6}\right)=0$ irrespective the flag. Thus, two of these 2-simplices are degenerate and contribute no volume. The only remaining term is

$$
\begin{aligned}
& =-\operatorname{Vol} \operatorname{simplex}\left\langle\underline{w}_{Y_{\bullet}}\left(h_{0}\right), \underline{w}_{Y_{\bullet}}\left(h_{4}\right)\right\rangle=-\frac{1}{2} \operatorname{det}\left(\begin{array}{ll}
\underline{w}_{1}^{\prime}\left(y^{-a}\right) & \underline{w}_{1}^{\prime}\left(x^{b}\right) \\
\underline{w}_{2}^{\prime}\left(y^{-a}\right) & \underline{w}_{2}^{\prime}\left(x^{b}\right)
\end{array}\right) \\
& =-\frac{1}{2} \operatorname{det}\left(\begin{array}{ll}
\underline{w}_{1}^{\prime}\left(\left(x^{-l} y^{-1}\right)^{a} \cdot x^{l a}\right) & \underline{w}_{1}^{\prime}\left(x^{b}\right) \\
\underline{w}_{2}^{\prime}\left(\left(x^{-l} y^{-1}\right)^{a} \cdot x^{l a}\right) & \underline{w}_{2}^{\prime}\left(x^{b}\right)
\end{array}\right)=-\frac{1}{2} \operatorname{det}\left(\begin{array}{cc}
-l a & -b \\
a & 0
\end{array}\right)=\frac{1}{2} a b .
\end{aligned}
$$

4.4. Conclusion. Combining the contribution of both flags, we obtain that the right-hand side of Theorem 3.17 is

$$
\left(\frac{1}{2} a b-\frac{1}{2} l a^{2}\right)+\left(\frac{1}{2} a b\right)=a b-\frac{1}{2} l a^{2} .
$$

We had already computed the volume of the Newton-Okounkov body in two different ways in Equation 4.7 as well as Equation 4.8 and (luckily!) we see that all values agree. It remains to compute the left-hand side of Theorem 3.17. Recall that this side originates from Proposition 1.5. We need to pick $m$ such that $m D$ is very ample, but on a toric variety a line bundle is ample if and only if it is very ample, so $m=1$ is enough. But then note that the trivializing equations are those listed in Equation 4.5, but they also mark the extremal points of the polytope in Figure 4.6, so this $i s$ the convex polytope in question. In total, Theorem 3.17 hence reads: For the standard lexicographic valuation $\underline{v}$ of a toric variety we have

$$
\begin{aligned}
& \text { Vol (convex hull } \left.\left(\underline{v}\left(y^{-a}\right) ; \underline{v}\left(x^{b-l a} y^{-a}\right) ; \underline{v}\left(x^{b}\right) ; 0\right)\right)= \\
& \qquad \frac{1}{2} \operatorname{det}\left(\begin{array}{ll}
\underline{w}_{1}\left(x^{b}\right) & \underline{w}_{1}\left(x^{b-l a} y^{-a}\right) \\
\underline{w}_{2}\left(x^{b}\right) & \underline{w}_{2}\left(x^{b-l a} y^{-a}\right)
\end{array}\right)-\frac{1}{2} \operatorname{det}\left(\begin{array}{ll}
\underline{w}_{1}\left(\left(x^{l} y\right)^{-a} \cdot x^{l a}\right) & \underline{w}_{1}\left(\left(x^{l} y\right)^{-a} \cdot x^{b}\right) \\
\underline{w}_{2}\left(\left(x^{l} y\right)^{-a} \cdot x^{l a}\right) & \underline{w}_{2}\left(\left(x^{l} y\right)^{-a} \cdot x^{b}\right)
\end{array}\right) \\
& \quad+\frac{1}{2} \operatorname{det}\left(\begin{array}{ll}
\underline{w}_{1}\left(\left(x^{l} y\right)^{-a} \cdot x^{l a}\right) & \underline{w}_{1}\left(x^{b}\right) \\
\underline{w}_{2}\left(\left(x^{l} y\right)^{-a} \cdot x^{l a}\right) & \underline{w}_{2}\left(x^{b}\right)
\end{array}\right)-\frac{1}{2} \operatorname{det}\left(\begin{array}{ll}
\underline{w}_{1}^{\prime}\left(y^{-a}\right) & \underline{w}_{1}^{\prime}\left(x^{b}\right) \\
\underline{w}_{2}^{\prime}\left(y^{-a}\right) & \underline{w}_{2}^{\prime}\left(x^{b}\right)
\end{array}\right) .
\end{aligned}
$$

This agrees with Equation 0.4 except that all the arguments are spelt out.

\section{Appendix A. Alternative proof of the main theorem}

In this appendix, we sketch an alternative proof of the main results. It removes any use of Milnor $K$-theory and uses residues instead. The proof is a little weaker because it only works in characteristic zero.

We only describe how to change the proof. Firstly, in Diagram 2.17 replace $H^{n}\left(X, \mathcal{K}_{n}^{\mathrm{M}}\right)$ by $H^{n}\left(X, \Omega_{X / \mathbb{C}}^{n}\right)$ and $\gamma$ by the differential logarithm

$$
\begin{aligned}
H^{*}\left(X, \mathcal{O}_{X}^{\times} \otimes \cdots \otimes \mathcal{O}_{X}^{\times}\right) & \longrightarrow H^{*}\left(X, \mathcal{K}_{*}^{\mathrm{M}}\right) \longrightarrow H^{*}\left(X, \Omega_{X / k}^{*}\right) \\
x_{1} \otimes \cdots \otimes x_{n} \longmapsto\left\{x_{1}, \ldots, x_{n}\right\} & \longmapsto \frac{\mathrm{d} x_{1}}{x_{1}} \wedge \cdots \wedge \frac{\mathrm{d} x_{n}}{x_{n}} .
\end{aligned}
$$

If we work over the complex numbers, the map $\tau$ of Diagram 2.17 then can be replaced by

$$
H^{n}\left(X, \Omega_{X / \mathbb{C}}^{n}\right) \cong H^{n, n}(X) \subseteq H^{2 n}(X, \mathbb{C}) \longrightarrow \mathbb{C},
$$


i.e. under the Dolbeault isomorphism we identify the cohomology group $H^{n}\left(X, \Omega_{X / \mathbb{C}}^{n}\right)$ with the $(n, n)$-classes in the top Betti cohomology. Doing these replacements is compatible with intersection theory, i.e. this still computes the same intersection number. However, even if $k$ is not the complex numbers, we can also just use the evaluation map of Serre duality, $H^{n}\left(X, \Omega_{X / k}^{n}\right) \rightarrow k$, using that $X / k$ is integral smooth proper over $k$. Now use the Cousin resolution of $\Omega_{X / k}^{n}$, Har66. Its terms are skyscraper sheaves of the shape

$$
U \mapsto \bigoplus_{x \in U^{(i)}} H_{x}^{p}\left(X, \Omega_{X / k}^{n}\right), \quad \text { where } H_{x}^{p}(-,-) \text { denotes local cohomology, }
$$

where $U^{(i)}$ denotes the set of points $x$ such that $\overline{\{x\}}$ has codimension $i$ in $X$. The 'algebraic partitions of unity' of [Bra13, §2.1] exist for such sheaves. Notably, Bra13, Lemma $2]$ applies. Then form the double complex comparing the Cech cohomology of $\Omega_{X / k}^{n}$ in a concrete open cover $\mathfrak{U}$ with the cohomology of the Cousin complex, imitating [Bra13, §2.2]. The boundary maps $\partial_{(-)}$get replaced by residue maps; the norm maps get replaced by trace maps. One proves the analogue of Proposition 3.9, where the left side gets replaced by $\operatorname{res}_{v_{n}} \cdots \operatorname{res}_{v_{1}}\left(\frac{\mathrm{d} f_{1}}{f_{1}} \wedge \cdots \wedge \frac{\mathrm{d} f_{n}}{f_{n}}\right)$ and "res $v$ " being the local residue map. This term can be understood as a Grothendieck residue symbol. Once this is all set up, adapt the proof of Theorem 3.15. If one runs this variant of the proof in chacteristic $p>0$, we only obtain equality in Theorem 3.15 modulo $p$, so this proof is strictly weaker than the one using Milnor $K$-groups.

\section{REFERENCES}

[AKL14] D. Anderson, A. Küronya, and V. Lozovanu, Okounkov bodies of finitely generated divisors, Int. Math. Res. Not. IMRN (2014), no. 9, 2343-2355. MR 3207370

[And13] D. Anderson, Okounkov bodies and toric degenerations, Math. Ann. 356 (2013), no. 3, 1183-1202. MR 3063911

[Blo74] S. Bloch, $K_{2}$ and algebraic cycles, Ann. of Math. (2) 99 (1974), 349-379. MR 0342514 (49 \#7260)

[Bra13] O. Braunling, Bad intersections and constructive aspects of the Bloch-Quillen formula, New York J. Math. 19 (2013), 545-564. MR 3104562

$\left[\mathrm{CFK}^{+} 17\right]$ C. Ciliberto, M. Farnik, A. Küronya, V. Lozovanu, J. Roé, and C. Shramov, Newton-Okounkov bodies sprouting on the valuative tree, Rend. Circ. Mat. Palermo (2) 66 (2017), no. 2, 161-194. MR 3694973

[CLS11] D. Cox, J. Little, and H. Schenck, Toric varieties, Graduate Studies in Mathematics, vol. 124, American Mathematical Society, Providence, RI, 2011. MR 2810322 (2012g:14094)

[Fu193] W. Fulton, Introduction to toric varieties, Annals of Mathematics Studies, vol. 131, Princeton University Press, Princeton, NJ, 1993, The William H. Roever Lectures in Geometry. MR 1234037 (94g:14028)

[GH94] P. Griffiths and J. Harris, Principles of algebraic geometry, Wiley Classics Library, John Wiley \& Sons, Inc., New York, 1994, Reprint of the 1978 original. MR 1288523

[Gil05] H. Gillet, $K$-theory and intersection theory, Handbook of $K$-theory. Vol. 1, 2, Springer, Berlin, 2005, pp. 235-293. MR 2181825 (2006h:14013)

[Gra78] D. Grayson, Products in K-theory and intersecting algebraic cycles, Invent. Math. 47 (1978), no. 1, 71-83. MR 0491685

[GS06] P. Gille and T. Szamuely, Central simple algebras and Galois cohomology, Cambridge Studies in Advanced Mathematics, vol. 101, Cambridge University Press, Cambridge, 2006. MR 2266528 (2007k:16033)

[Har66] R. Hartshorne, Residues and duality, Lecture notes of a seminar on the work of A. Grothendieck, given at Harvard 1963/64. With an appendix by P. Deligne. Lecture Notes in Mathematics, No. 20, Springer-Verlag, Berlin, 1966. MR 0222093 (36 \#5145)

[Ker09] M. Kerz, The Gersten conjecture for Milnor K-theory, Invent. Math. 175 (2009), no. 1, 1-33. MR 2461425 (2010i:19004) 
[KK12] K. Kaveh and A. G. Khovanskii, Newton-Okounkov bodies, semigroups of integral points, graded algebras and intersection theory, Ann. of Math. (2) 176 (2012), no. 2, 925-978. MR 2950767

[KL18] A. Küronya and V. Lozovanu, Local positivity of linear series on surfaces, Algebra Number Theory 12 (2018), no. 1, 1-34. MR 3781431

[KLM12] A. Küronya, V. Lozovanu, and C. Maclean, Convex bodies appearing as Okounkov bodies of divisors, Adv. Math. 229 (2012), no. 5, 2622-2639. MR 2889138

[Laz04] R. Lazarsfeld, Positivity in algebraic geometry. I, Ergebnisse der Mathematik und ihrer Grenzgebiete. 3. Folge. A Series of Modern Surveys in Mathematics [Results in Mathematics and Related Areas. 3rd Series. A Series of Modern Surveys in Mathematics], vol. 48, Springer-Verlag, Berlin, 2004, Classical setting: line bundles and linear series. MR 2095471

[LM09] R. Lazarsfeld and M. Mustaţă, Convex bodies associated to linear series, Ann. Sci. Éc. Norm. Supér. (4) 42 (2009), no. 5, 783-835. MR 2571958

[Mil70] J. Milnor, Algebraic K-theory and quadratic forms, Invent. Math. 9 (1969/1970), 318-344. MR 0260844 (41 \#5465)

[Oko96] A. Okounkov, Brunn-Minkowski inequality for multiplicities, Invent. Math. 125 (1996), no. 3, 405-411. MR 1400312

[Oko03] — Why would multiplicities be log-concave?, The orbit method in geometry and physics (Marseille, 2000), Progr. Math., vol. 213, Birkhäuser Boston, Boston, MA, 2003, pp. 329-347. MR 1995384

[Par83] A. N. Parshin, Chern classes, adèles and L-functions, J. Reine Angew. Math. 341 (1983), 174-192. MR 697316 (85c:14015)

[PF99] A. Parshin and T. Fimmel, Introduction to higher adelic theory (draft), unpublished, 1999.

[Ros96] M. Rost, Chow groups with coefficients, Doc. Math. 1 (1996), No. 16, 319-393 (electronic). MR 1418952 (98a:14006)

[ZS75] O. Zariski and P. Samuel, Commutative algebra. Vol. II, Springer-Verlag, New York-Heidelberg, 1975, Reprint of the 1960 edition, Graduate Texts in Mathematics, Vol. 29. MR 0389876

Mathematical Institute, University of Freiburg, Ernst-Zermelo-Strasse 1, 79104 Freiburg im Breisgau, Germany 